\title{
An N-Aryl-Substituted Oxazolidinone-Containing Ketone-Catalyzed Asymmetric Epoxidation Using Hydrogen Peroxide as Primary Oxidant
}

\author{
Christopher P. Burke, Lianhe Shu, and Yian Shi* \\ Department of Chemistry \\ Colorado State University \\ Fort Collins, CO 80523 \\ Phone: 970-491-7424 \\ Fax: 970-491-1801 \\ Email: yian@lamar.colostate.edu
}

\section{Supporting Information}

\section{Table of Contents}

HPLC and GC data for the determination of the ee's 
The chromatograms for the determination of enantiomeric excess of the epoxides from the asymmetric epoxidation of olefins catalyzed by ketone $\mathbf{2}$.

Table 2, Entry 1

$\mathrm{P}_{\mathrm{Ph}}$

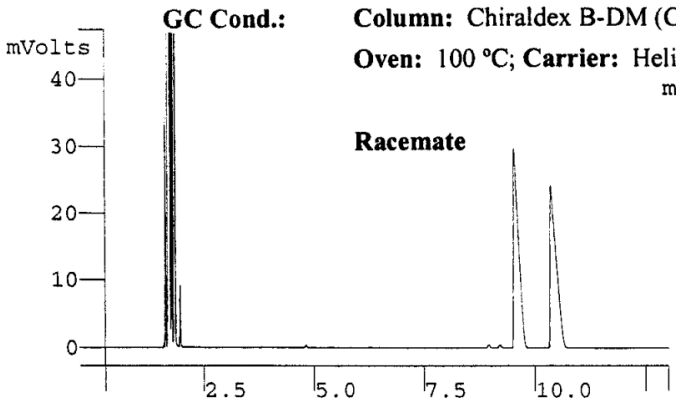

Column: Chiraldex B-DM (Cat. No. 77023), Adv. Separation Technologies, Inc

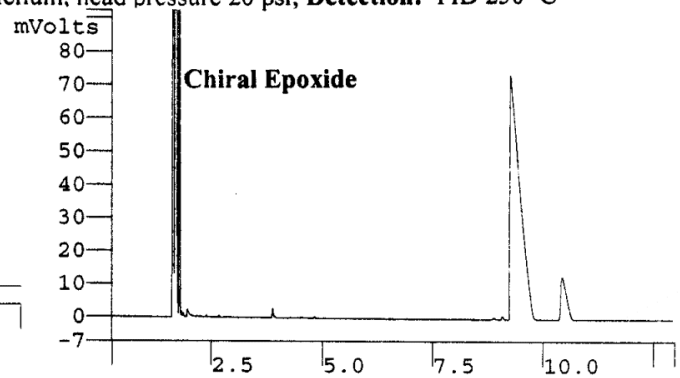

\begin{tabular}{cccccccccc}
$\begin{array}{c}\text { Peak } \\
\text { No }\end{array}$ & $\begin{array}{c}\text { Peak } \\
\text { Name }\end{array}$ & $\begin{array}{c}\text { Result } \\
0\end{array}$ & $\begin{array}{c}\text { Ret. } \\
\text { Time } \\
(\mathbf{m i n})\end{array}$ & $\begin{array}{c}\text { Area } \\
\text { (counts) }\end{array}$ & $\begin{array}{c}\text { Peak } \\
\text { No }\end{array}$ & $\begin{array}{c}\text { Peak } \\
\text { Name }\end{array}$ & $\begin{array}{c}\text { Result } \\
0\end{array}$ & $\begin{array}{c}\text { Ret. } \\
\text { Time } \\
\text { (min) }\end{array}$ & $\begin{array}{c}\text { Minutes } \\
\text { Area } \\
\text { (counts) }\end{array}$ \\
\hline 1 \\
\hline 2
\end{tabular}<smiles>CC1(C)OC1c1ccccc1</smiles>

GC Cond.: Column: Chiraldex B-DM (Cat. No. 77023), Adv. Separation Technologies, Inc.

Oven: $110^{\circ} \mathrm{C}$; Carrier: Helium, head pressure $20 \mathrm{psi}$; Detection: FID $250^{\circ} \mathrm{C}$
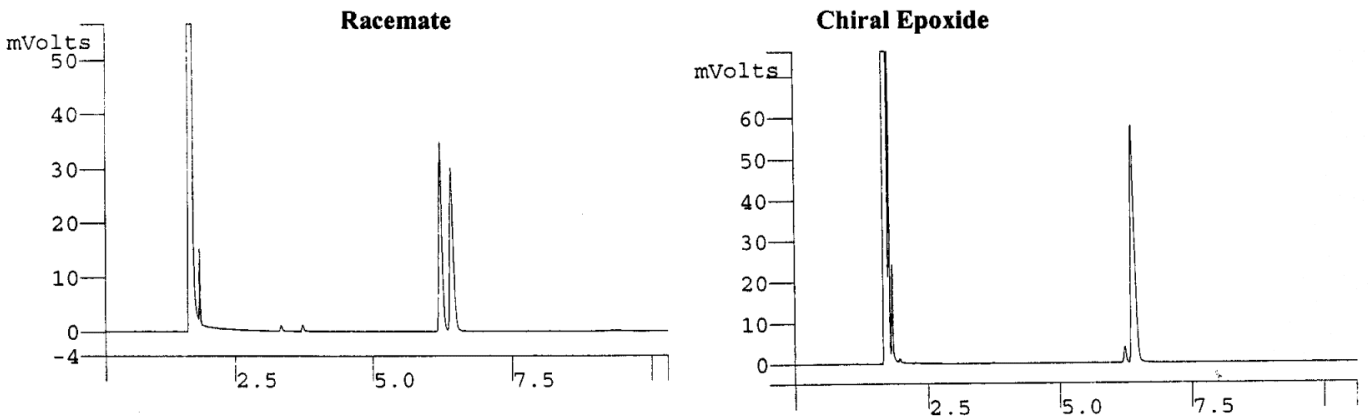

\begin{tabular}{|c|c|c|c|c|}
\hline $\begin{array}{c}\text { Peak } \\
\text { No }\end{array}$ & $\begin{array}{l}\text { Peak } \\
\text { Name }\end{array}$ & $\begin{array}{c}\text { Result } \\
0\end{array}$ & $\begin{array}{l}\text { Ret. } \\
\text { Time } \\
\text { (min) }\end{array}$ & $\begin{array}{l}\text { Minutes } \\
\text { Area } \\
\text { (counts) }\end{array}$ \\
\hline 1 & & 50.2361 & 6.204 & 129911 \\
\hline 2 & & 49.7639 & 6.403 & 128689 \\
\hline & Totals & 100.0000 & & 258600 \\
\hline
\end{tabular}

\begin{tabular}{|c|c|c|c|c|}
\hline $\begin{array}{c}\text { Peak } \\
\text { No }\end{array}$ & $\begin{array}{c}\text { Peak } \\
\text { Name }\end{array}$ & $\begin{array}{c}\text { Result } \\
0\end{array}$ & $\begin{array}{l}\text { Ret. } \\
\text { Time } \\
\text { (min) }\end{array}$ & $\begin{array}{l}\text { Minutes } \\
\text { Area } \\
\text { (counts) }\end{array}$ \\
\hline 1 & & 3.8117 & 6.226 & 11569 \\
\hline 2 & & 96.1883 & 6.358 & 291935 \\
\hline & Totals & 100.0000 & & 303504 \\
\hline
\end{tabular}


Table 2, Entry 3

$\mathrm{Ph}$ Kiph

HPLC Cond.: Column: Chiralcel OD (Column No. OD00CE-DL010), Chiral Technologies, Inc. Eluent: Hexanes/IPA (90/10); Flow Rate: 1.0mL/min; Detection: UV $220 \mathrm{~nm}$
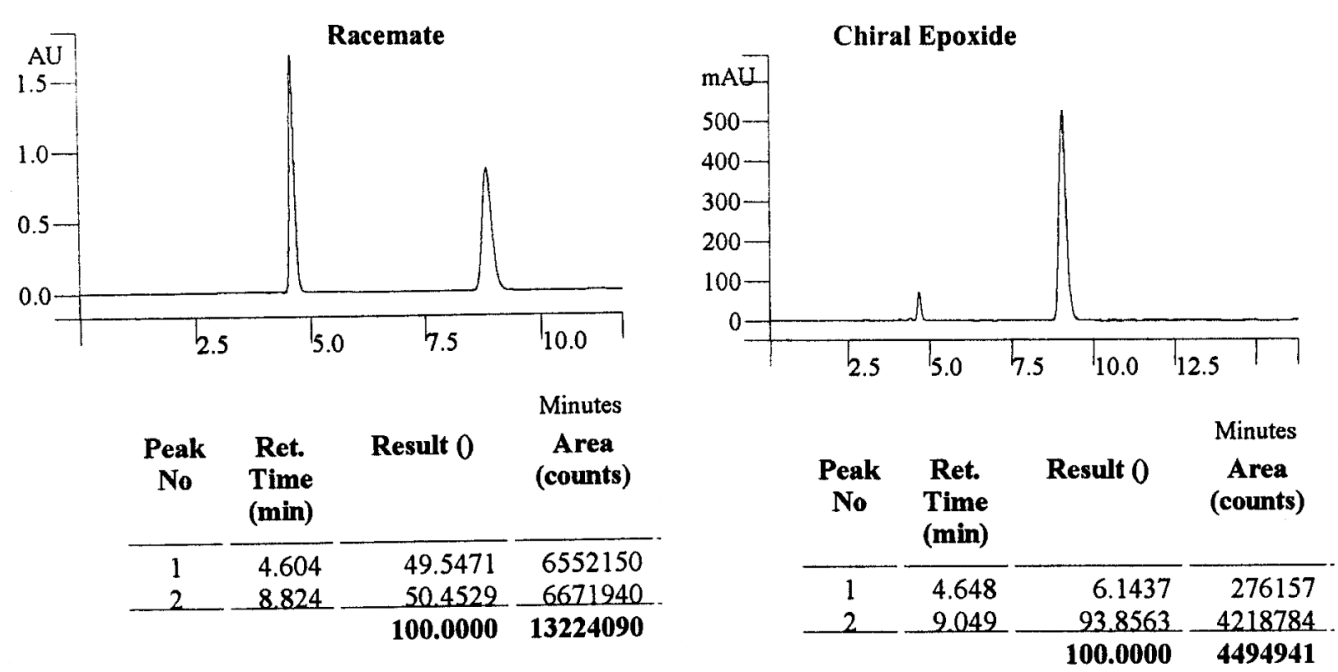

Table 2, Entry 4<smiles>O=[N+]([O-])c1cccc(C2CO2)c1</smiles>

GC Cond.: Column: Chiraldex B-DM (Cat. No. 77023), Adv. Separation Technologies, Inc. Oven: $60^{\circ} \mathrm{C}$; Carrier: Helium, head pressure 25 psi; Detection: FID $250^{\circ} \mathrm{C}$
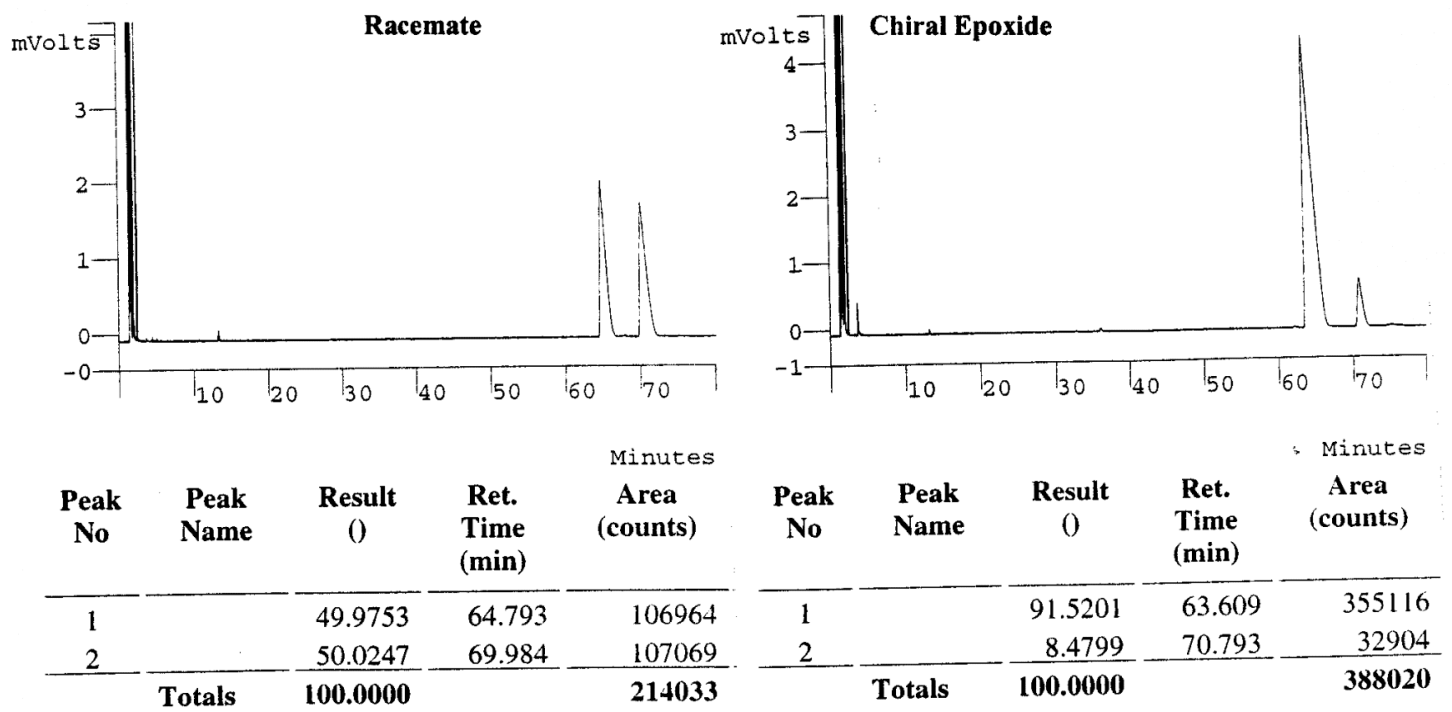
Table 2, Entry 5<smiles>COc1cccc(C2CO2)c1</smiles>

HPLC Cond.: Column: Chiralcel OD (Column No. OD00CE-DL010), Chiral Technologies, Inc. Eluent: Hexanes/IPA (95/5); Flow Rate: $1.0 \mathrm{~mL} / \mathrm{min}$; Detection: UV $270 \mathrm{~nm}$
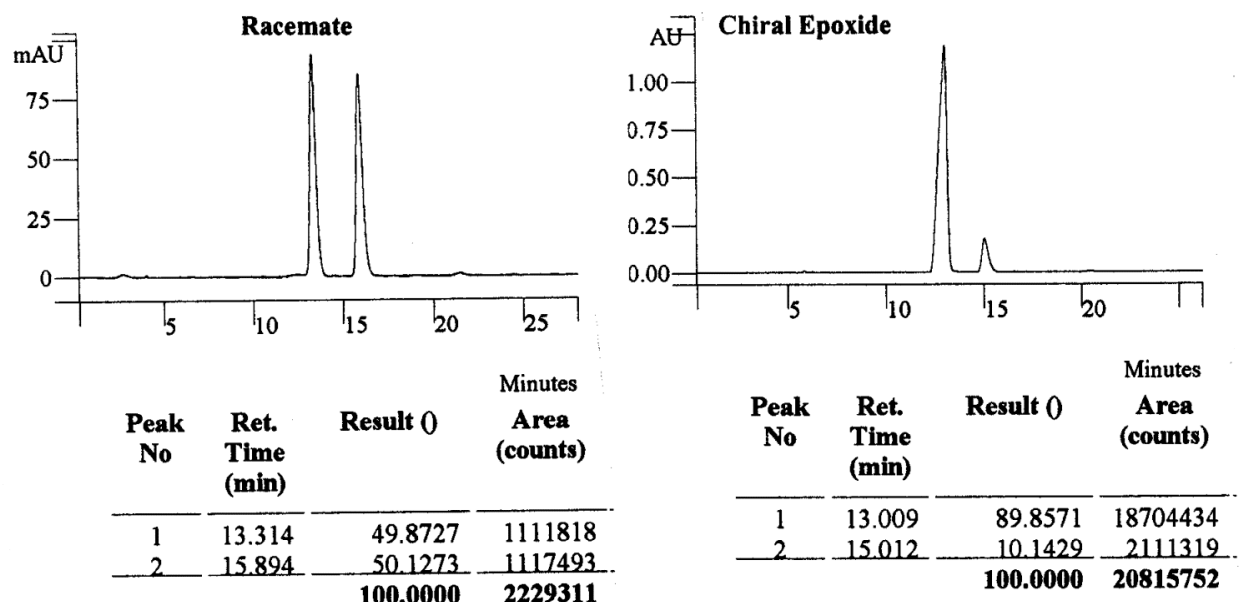

Table 2, Entry 6<smiles>CC1(C)Oc2ccc([N+](=O)[O-])cc2C2OC21</smiles>

HPLC Cond.: Column: Chiralcel OD (Column No. OD00CE-DL010), Chiral Technologies, Inc. Eluent: Hexanes/IPA (94/6); Flow Rate: $1.0 \mathrm{~mL} / \mathrm{min}$; Detection: UV $270 \mathrm{~nm}$
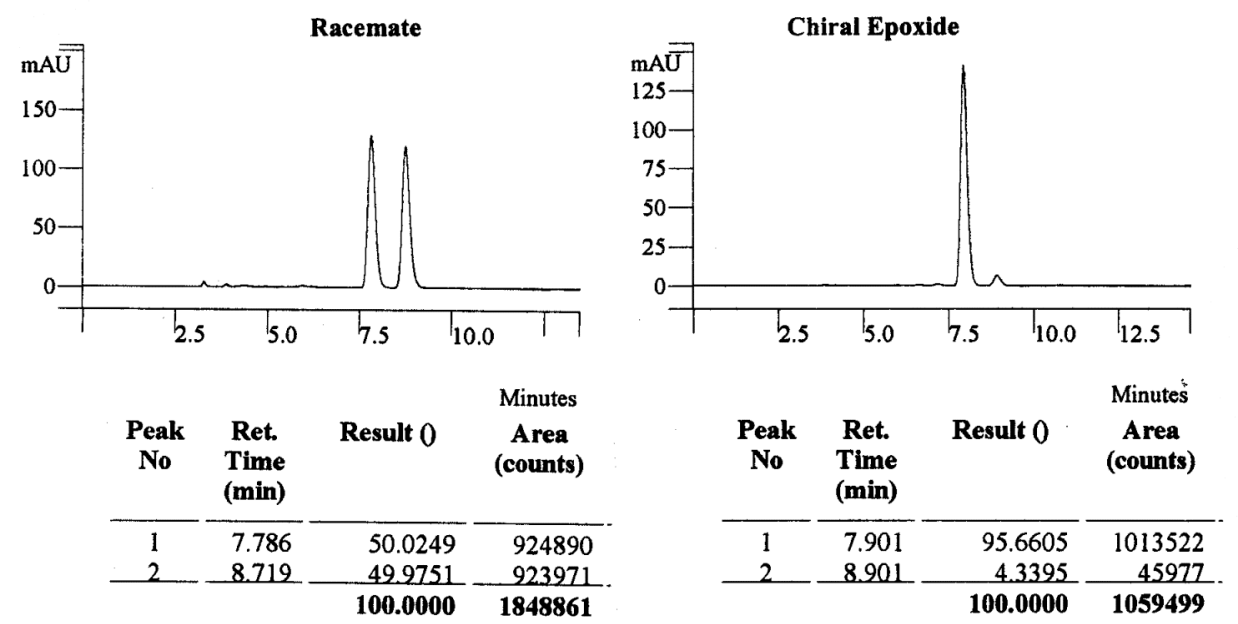


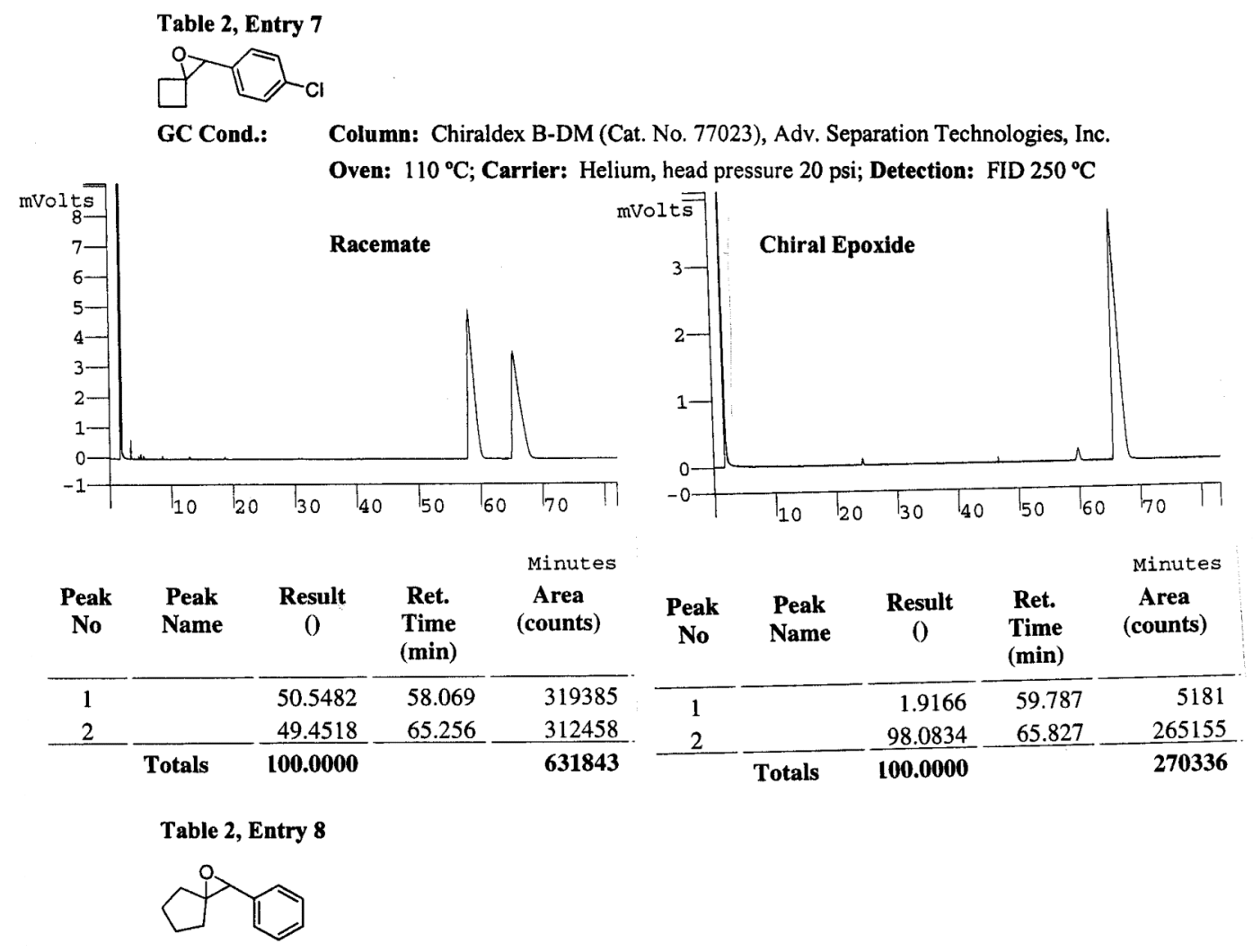

GC Cond.: Column: Chiraldex B-DM (Cat. No. 77023), Adv. Separation Technologies, Inc.

Oven: $100^{\circ} \mathrm{C}$; Carrier: Helium, head pressure 20 psi; Detection: FID $250^{\circ} \mathrm{C}$
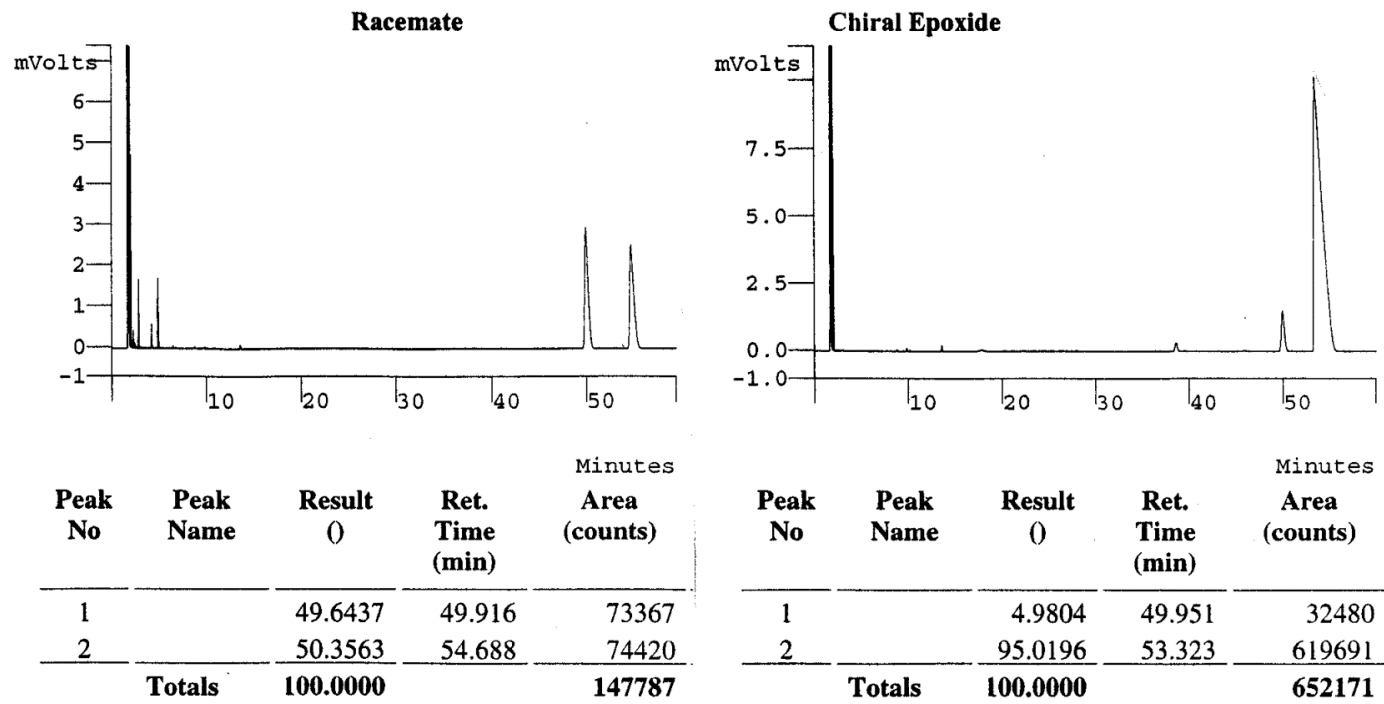

\begin{tabular}{|c|c|c|c|c|}
\hline $\begin{array}{c}\text { Peak } \\
\text { No }\end{array}$ & $\begin{array}{l}\text { Peak } \\
\text { Name }\end{array}$ & $\begin{array}{c}\text { Result } \\
0\end{array}$ & $\begin{array}{l}\text { Ret. } \\
\text { Time } \\
\text { (min) }\end{array}$ & $\begin{array}{l}\text { Minutes } \\
\text { Area } \\
\text { (counts) }\end{array}$ \\
\hline 1 & & 4.9804 & 49.951 & 32480 \\
\hline 2 & & 95.0196 & 53.323 & 619691 \\
\hline & Totals & 100.0000 & & 652171 \\
\hline
\end{tabular}


Table 2, Entry 9<smiles>CC1OC1C#C[Pb]</smiles>

HPLC Cond.: Column: Chiralcel OD (Column No. OD00CE-DL010), Chiral Technologies, Inc. Eluent: Hexanes/IPA (96/4); Flow Rate: $1.0 \mathrm{~mL} / \mathrm{min}$; Detection: UV $254 \mathrm{~nm}$
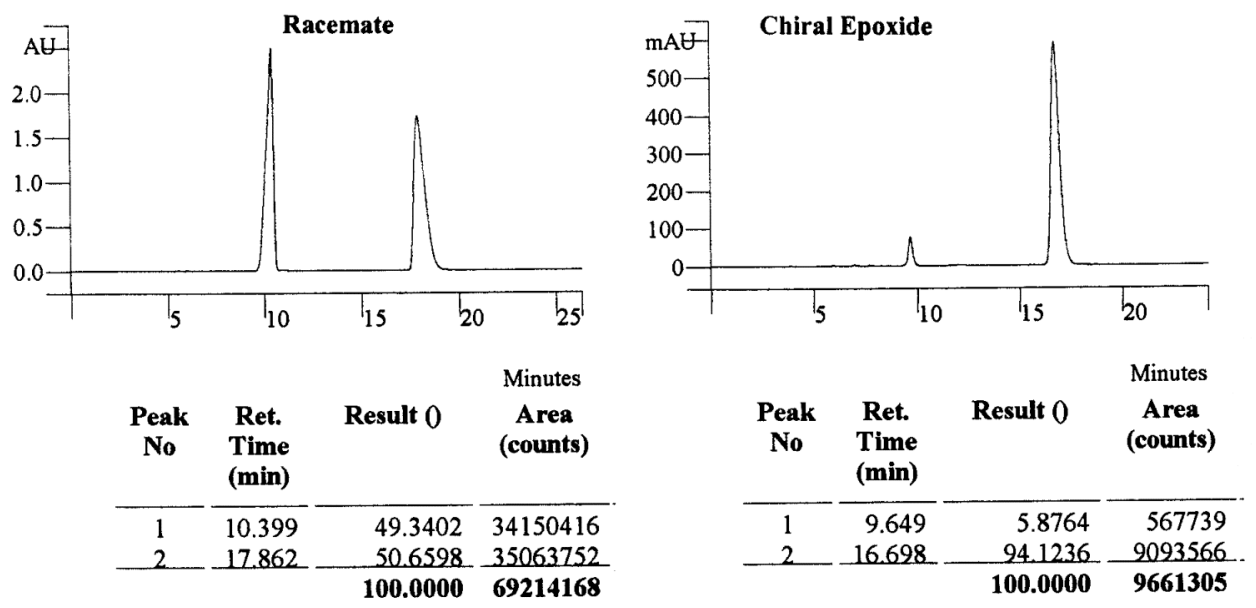

Table 2, Entry 10<smiles></smiles>

GC Cond.: Column: Chiraldex B-DM (Cat. No. 77023), Adv. Separation Technologies, Inc. Oven: $100^{\circ} \mathrm{C}$; Carrier: Helium, head pressure 20 psi; Detection: FID $250^{\circ} \mathrm{C}$
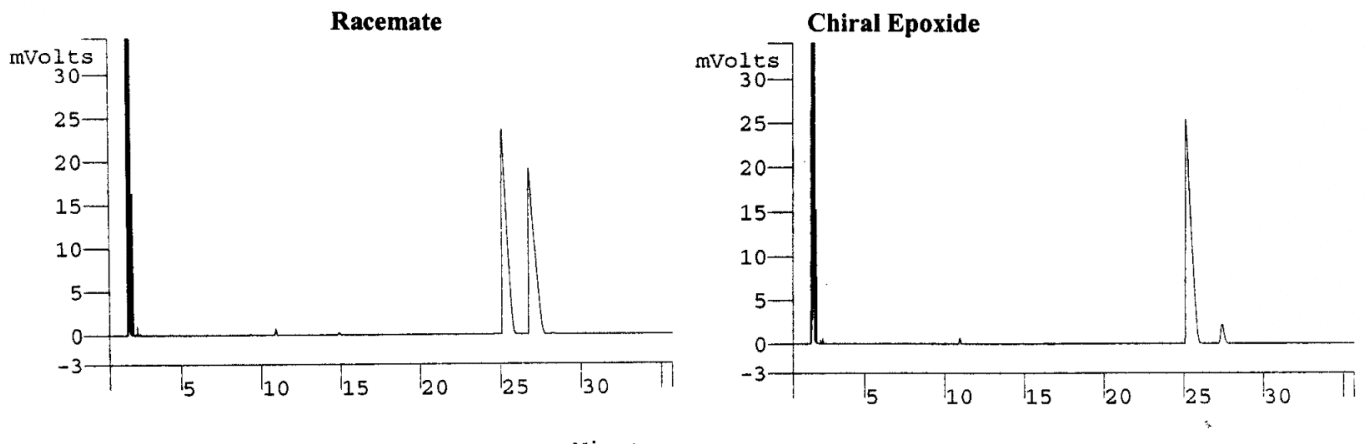

\begin{tabular}{|c|c|c|c|c|}
\hline $\begin{array}{c}\text { Peak } \\
\text { No }\end{array}$ & $\begin{array}{l}\text { Peak } \\
\text { Name }\end{array}$ & $\begin{array}{c}\text { Result } \\
(0\end{array}$ & $\begin{array}{l}\text { Ret. } \\
\text { Time } \\
\text { (min) }\end{array}$ & $\begin{array}{l}\text { Minutes } \\
\text { Area } \\
\text { (counts) }\end{array}$ \\
\hline 1 & & 50.1930 & 25.129 & 495145 \\
\hline 2 & & 49.8070 & 26.796 & 491338 \\
\hline & otals & 100.0000 & & 986483 \\
\hline
\end{tabular}

\begin{tabular}{|c|c|c|c|c|}
\hline $\begin{array}{c}\text { Peak } \\
\text { No }\end{array}$ & $\begin{array}{c}\text { Peak } \\
\text { Name }\end{array}$ & $\begin{array}{c}\text { Result } \\
0\end{array}$ & $\begin{array}{l}\text { Ret. } \\
\text { Time } \\
\text { (min) }\end{array}$ & $\begin{array}{c}\text { Area } \\
\text { (counts) }\end{array}$ \\
\hline 1 & & 94.9904 & 25.139 & 596376 \\
\hline 2 & & 5.0096 & 27.409 & 31452 \\
\hline & otals & 100.0000 & & 627828 \\
\hline
\end{tabular}


Table 2, Entry 11

Onotвs

GC Cond.: Column: Chiraldex B-DM (Cat. No. 77023), Adv. Separation Technologies, Inc.

Oven: $110^{\circ} \mathrm{C}$; Carrier: Helium, head pressure 20 psi; Detection: FID $250^{\circ} \mathrm{C}$
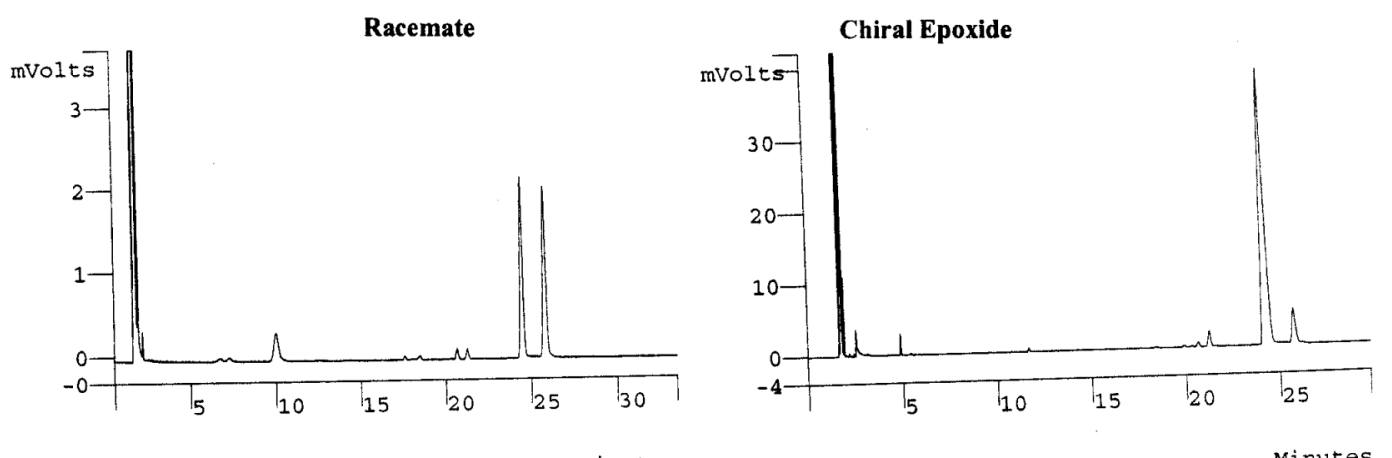

\begin{tabular}{ccccc}
$\begin{array}{c}\text { Peak } \\
\text { No }\end{array}$ & $\begin{array}{c}\text { Peak } \\
\text { Name }\end{array}$ & $\begin{array}{c}\text { Result } \\
()\end{array}$ & $\begin{array}{c}\text { Ret. } \\
\text { Time } \\
\text { (min) }\end{array}$ & $\begin{array}{c}\text { Area } \\
\text { (counts) }\end{array}$ \\
\hline 1 & & $\begin{array}{c}49.8737 \\
2\end{array} \frac{24.469}{50.1263}$ & 25.791 & 24316 \\
\hline Totals & $\frac{24439}{\mathbf{1 0 0 . 0 0 0 0}}$ & & 48755
\end{tabular}

\begin{tabular}{|c|c|c|c|c|}
\hline $\begin{array}{c}\text { Peak } \\
\text { No }\end{array}$ & $\begin{array}{c}\text { Peak } \\
\text { Name }\end{array}$ & $\begin{array}{c}\text { Result } \\
0\end{array}$ & $\begin{array}{l}\text { Ret. } \\
\text { Time } \\
(\text { min) }\end{array}$ & $\begin{array}{l}\text { Minutes } \\
\text { Area } \\
\text { (counts) }\end{array}$ \\
\hline 1 & & 91.1583 & 24.099 & 623515 \\
\hline 2 & & 8.8417 & 25.726 & 60476 \\
\hline & Totals & 100.0000 & & 683991 \\
\hline
\end{tabular}




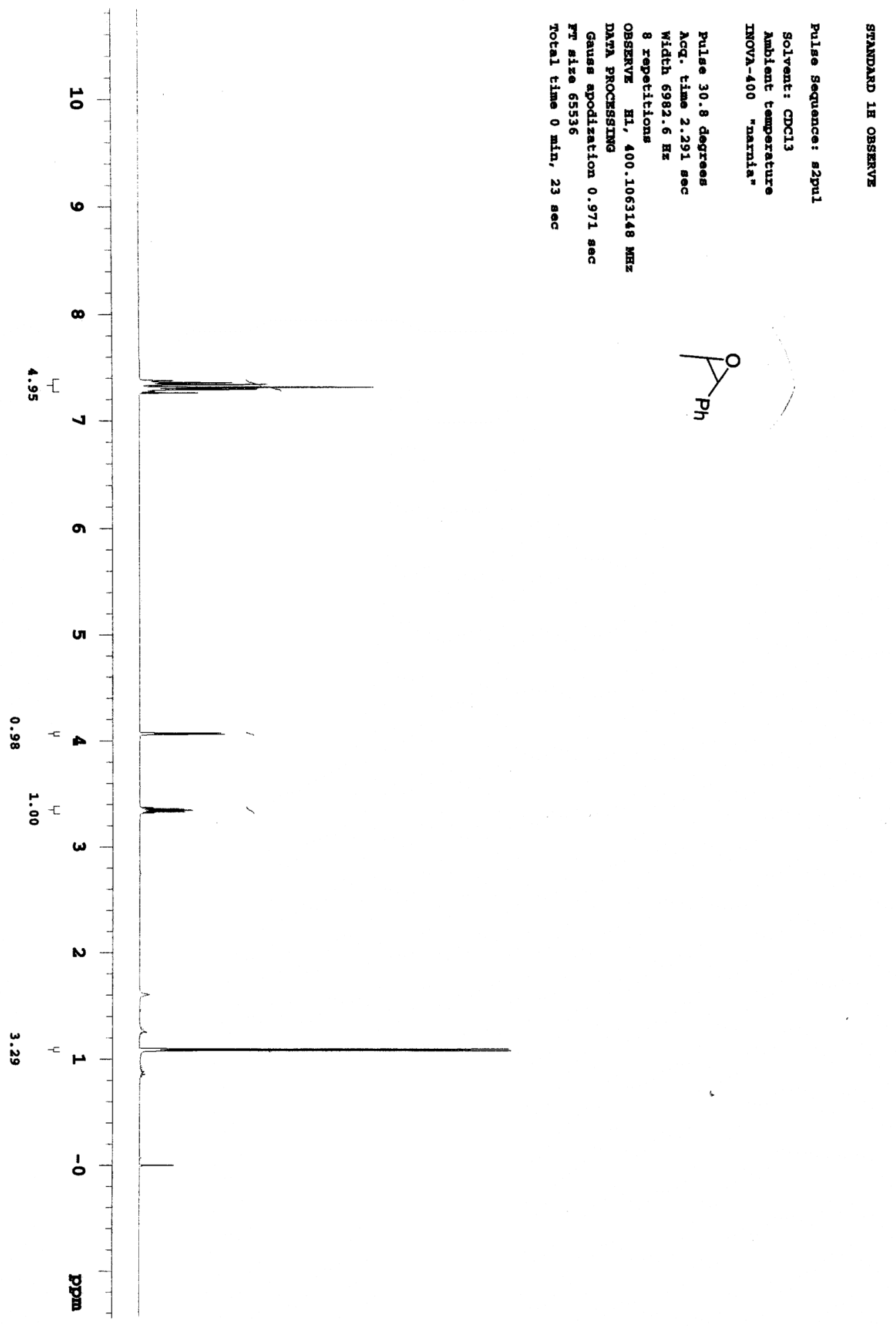




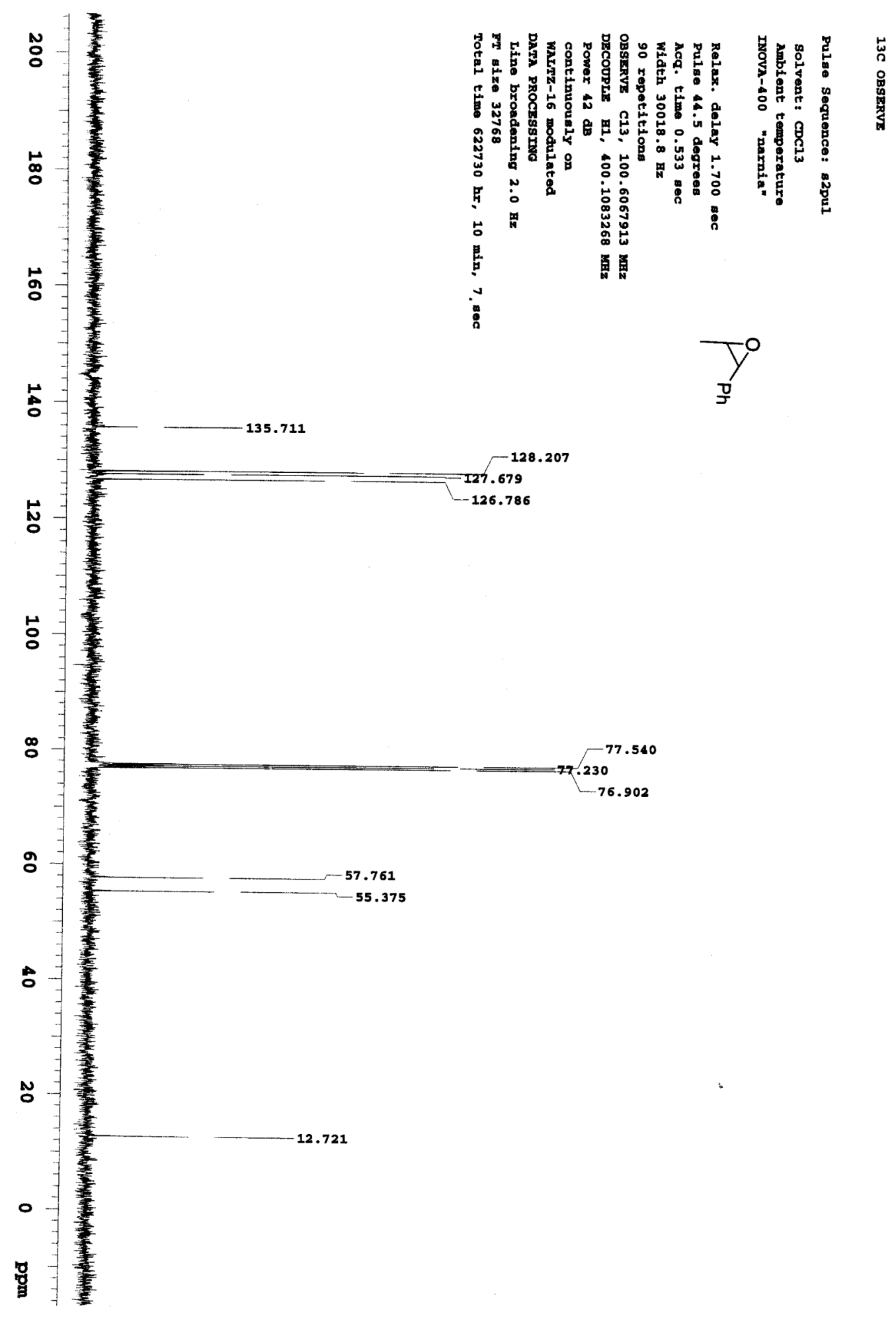




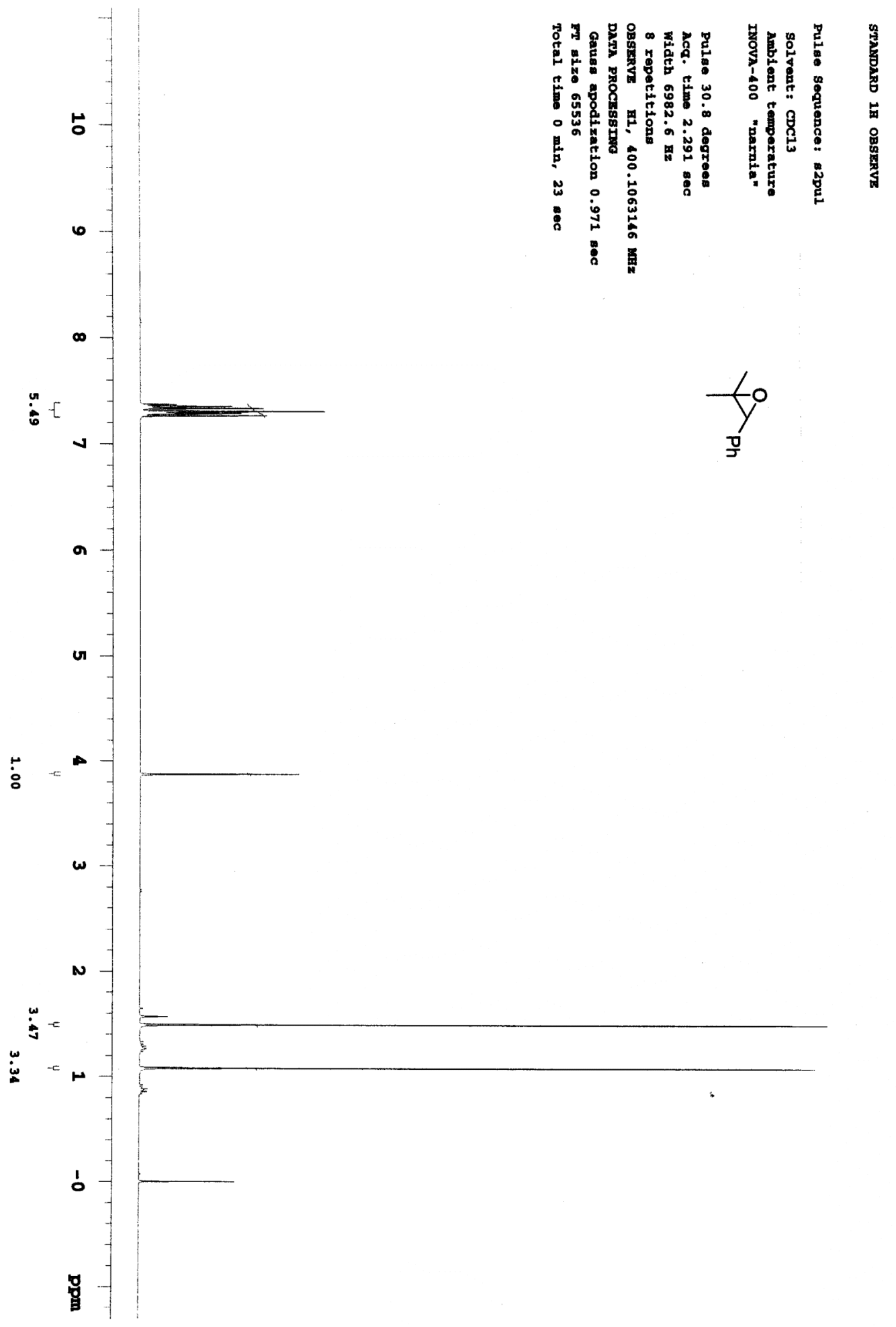




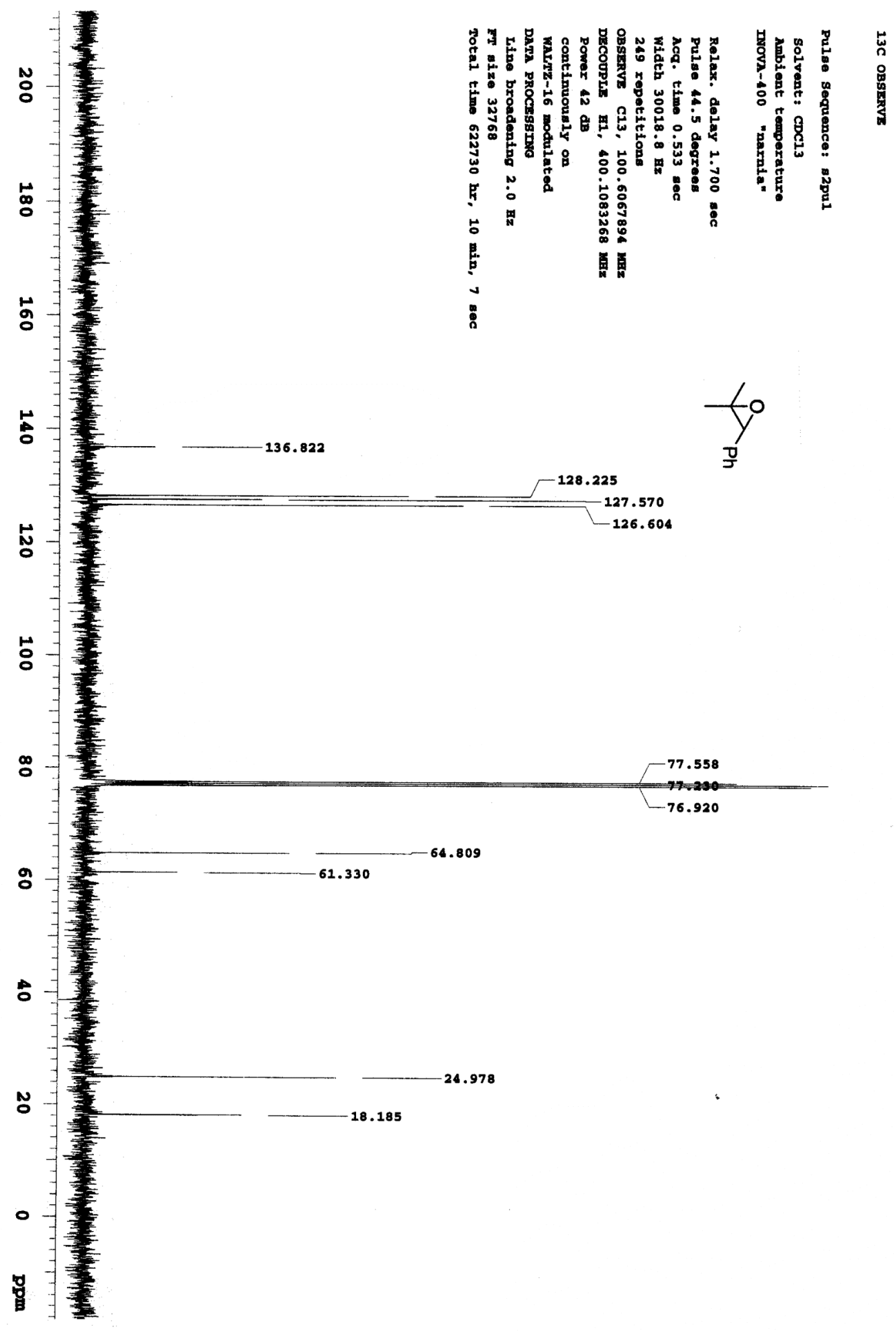




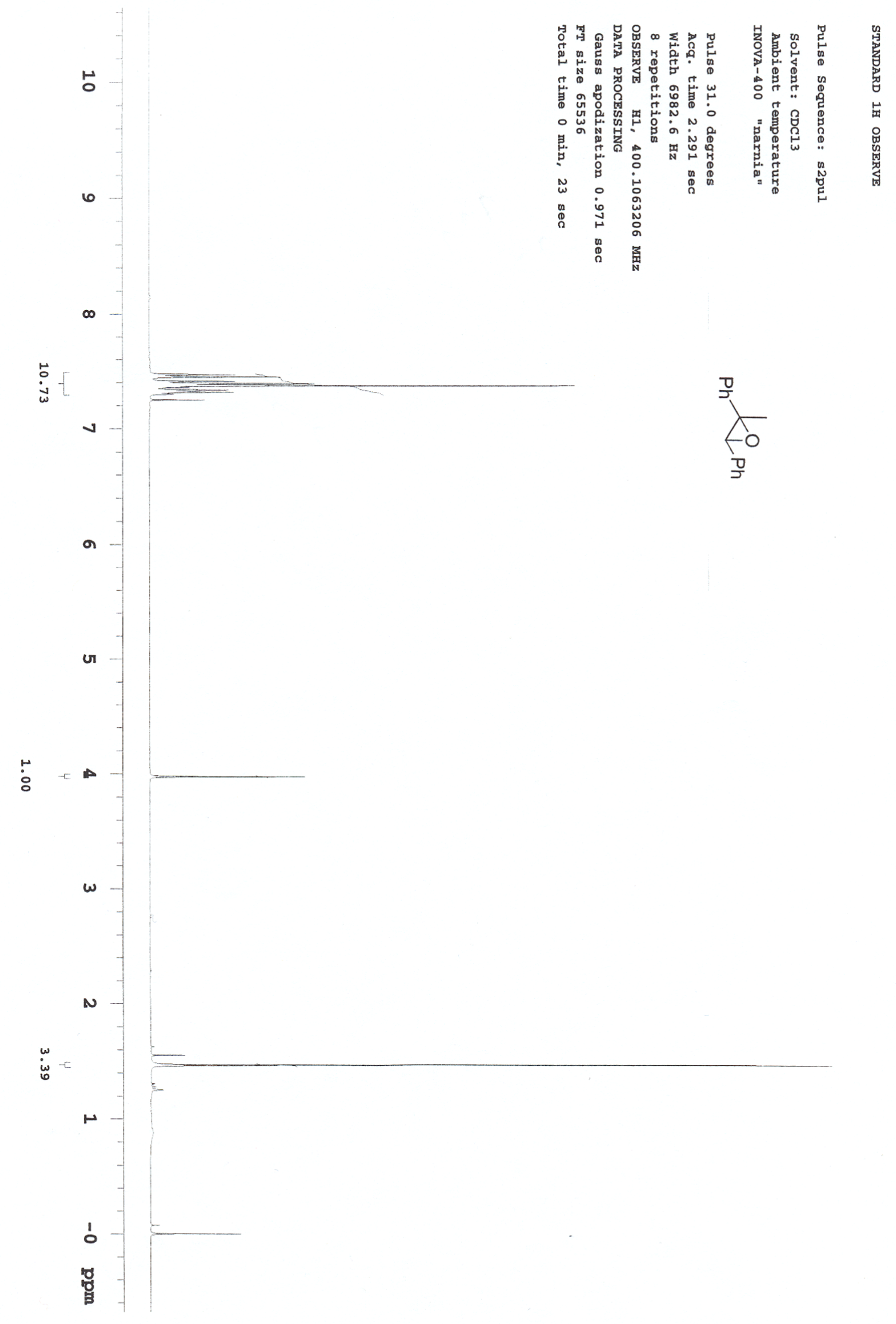




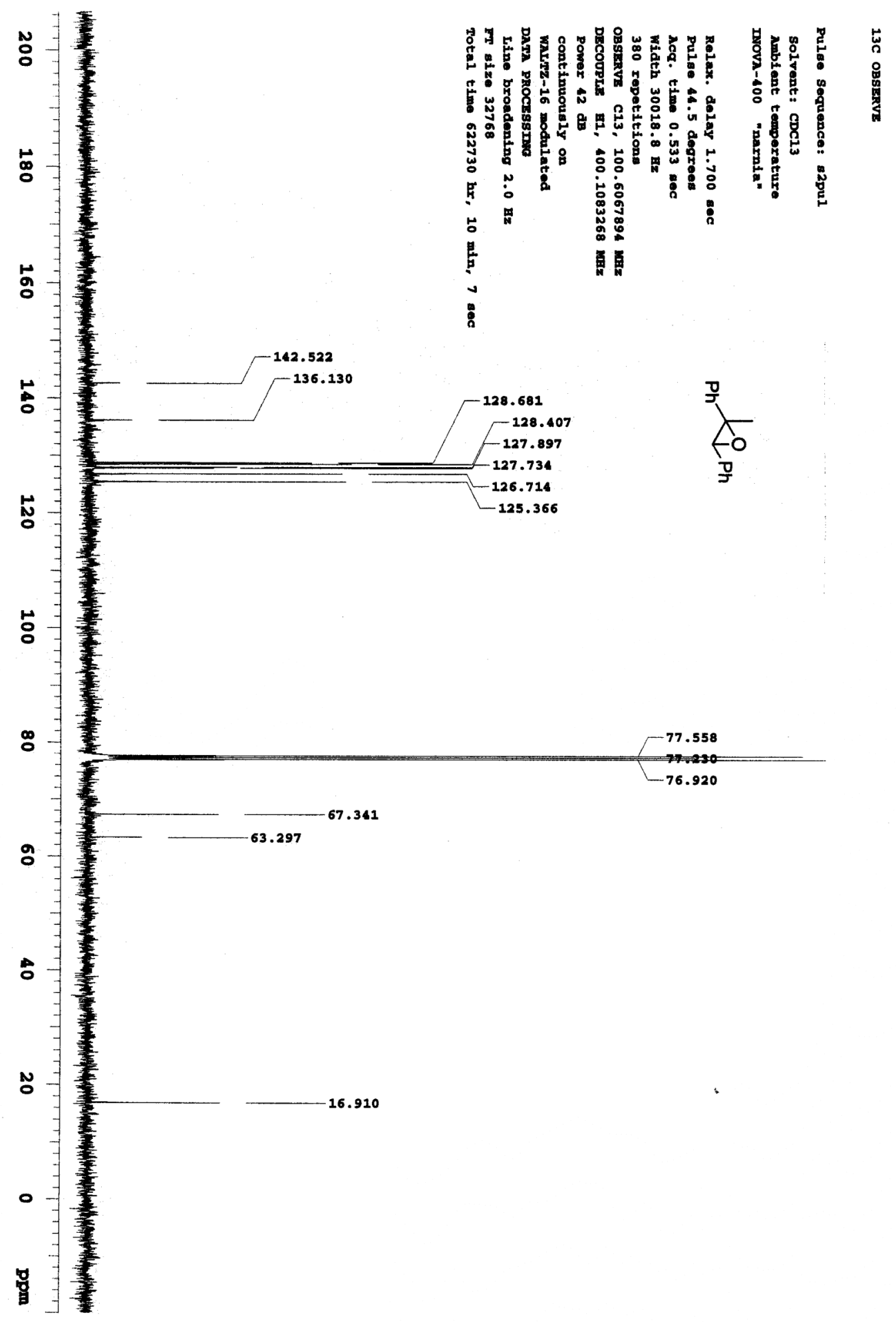




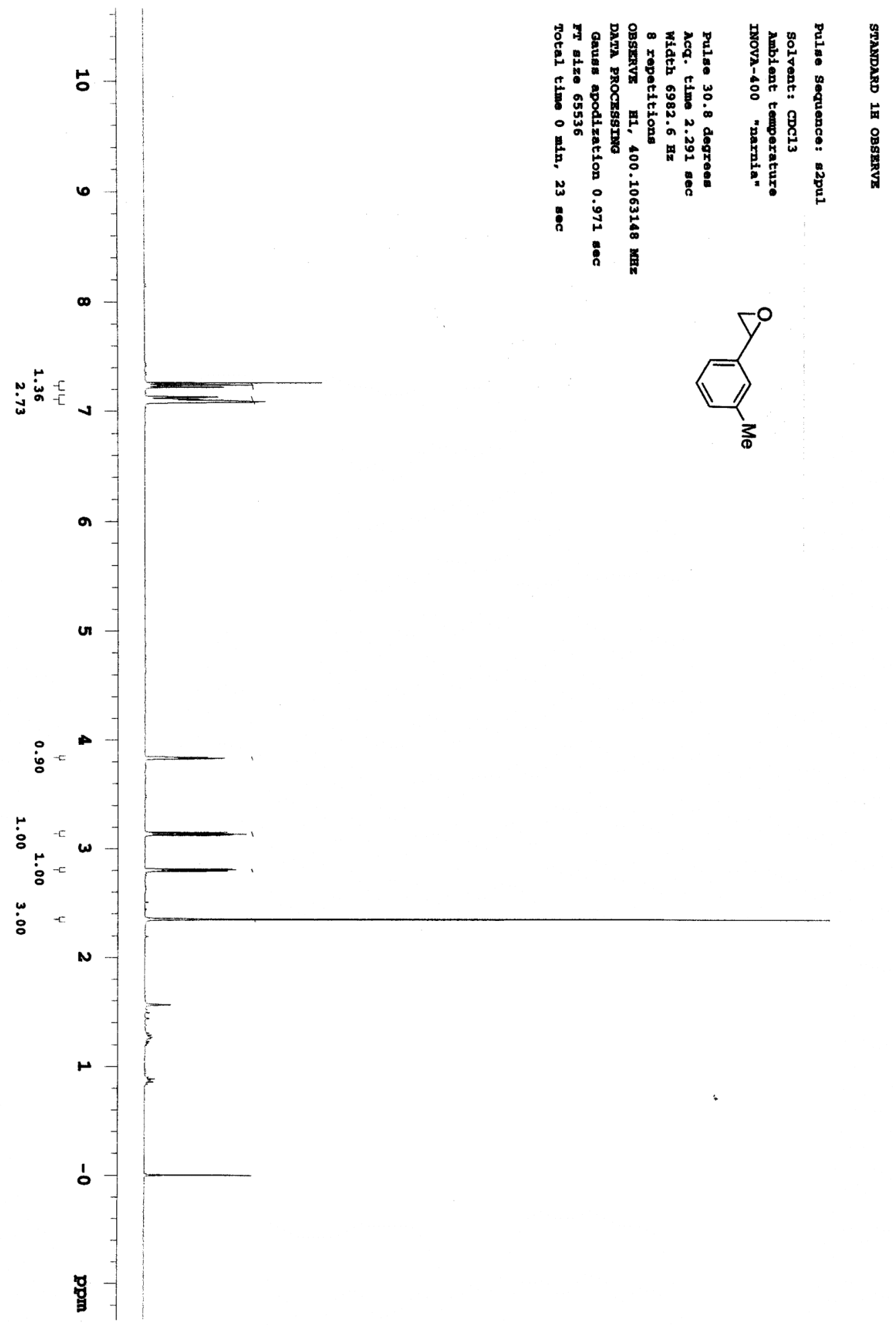




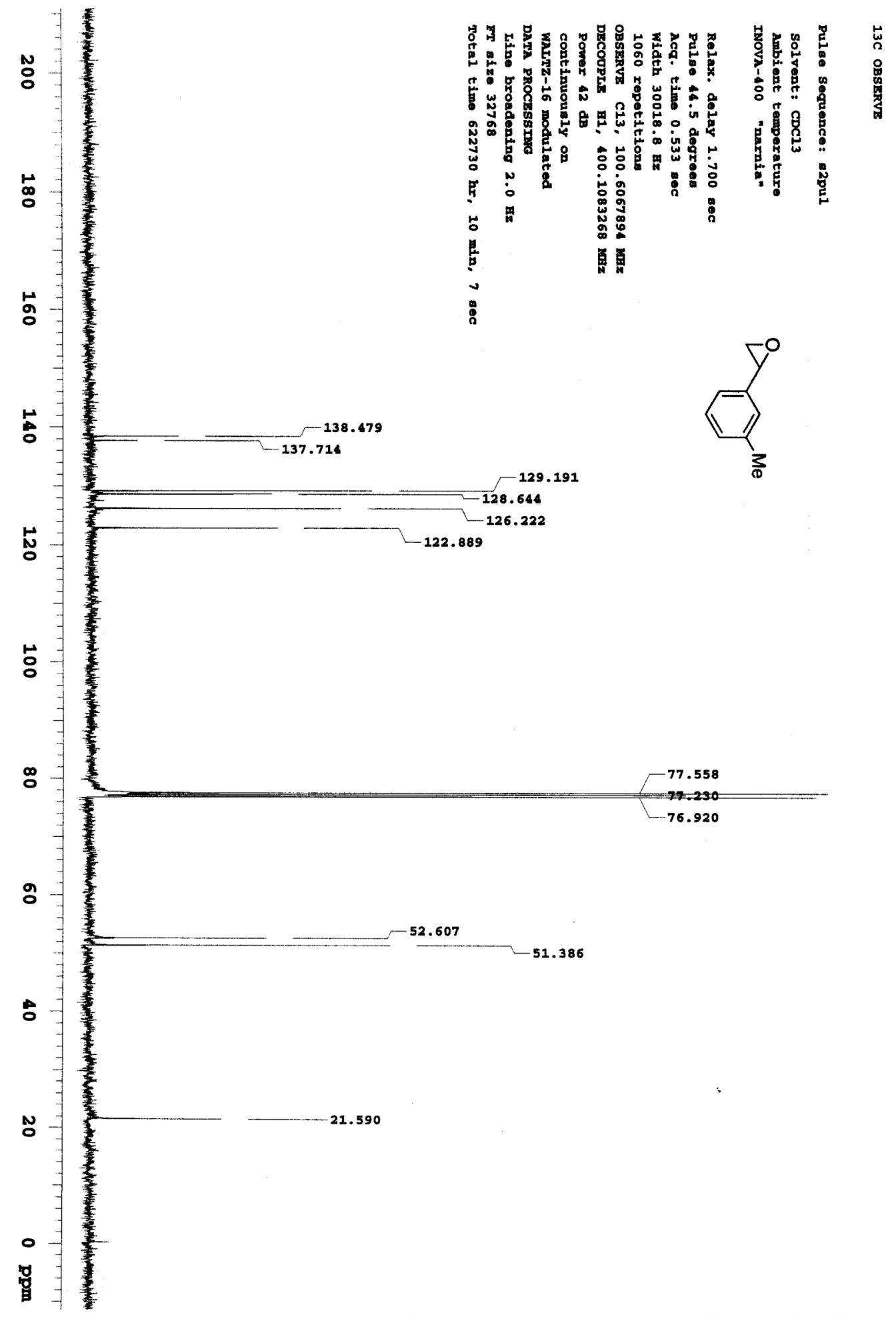




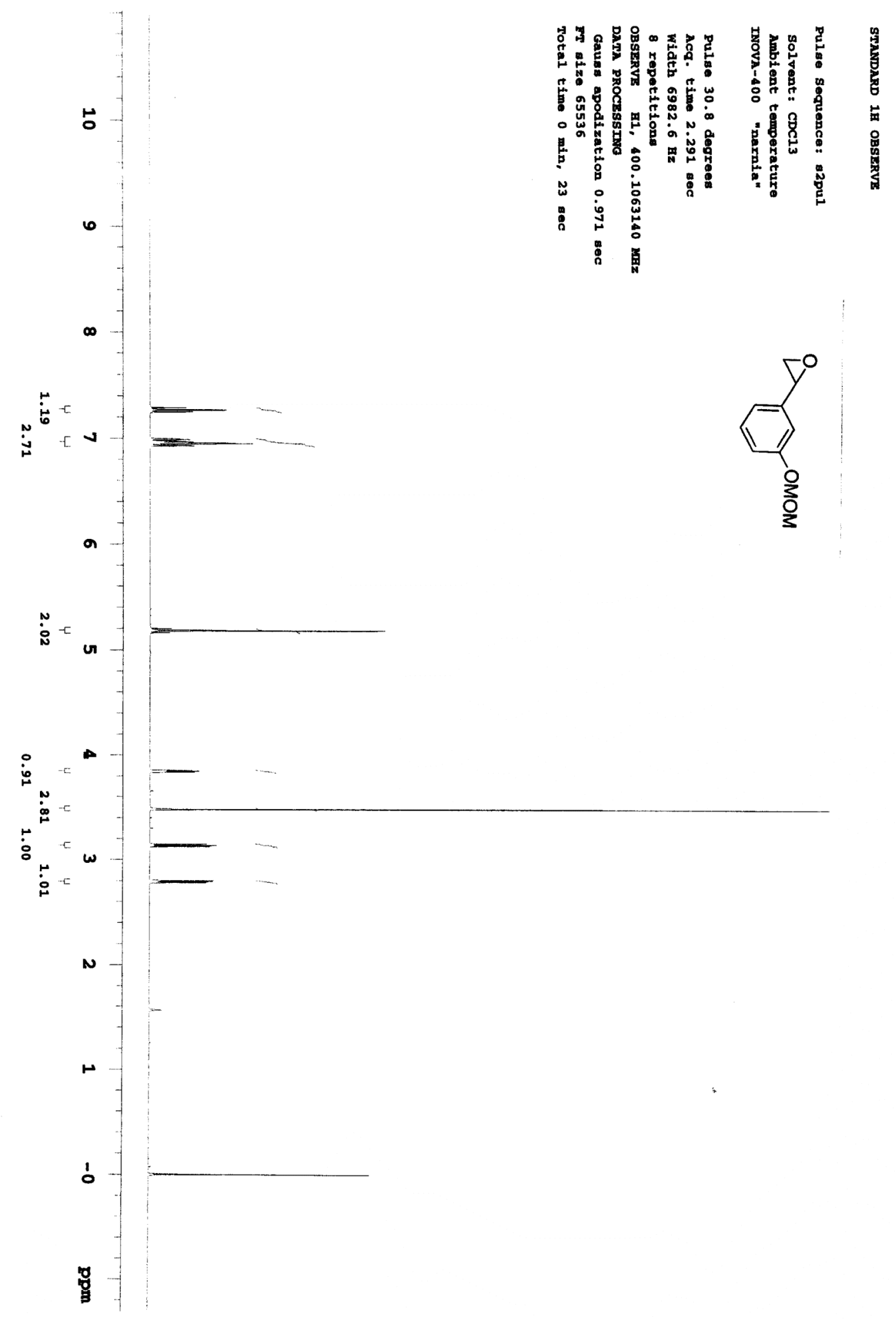




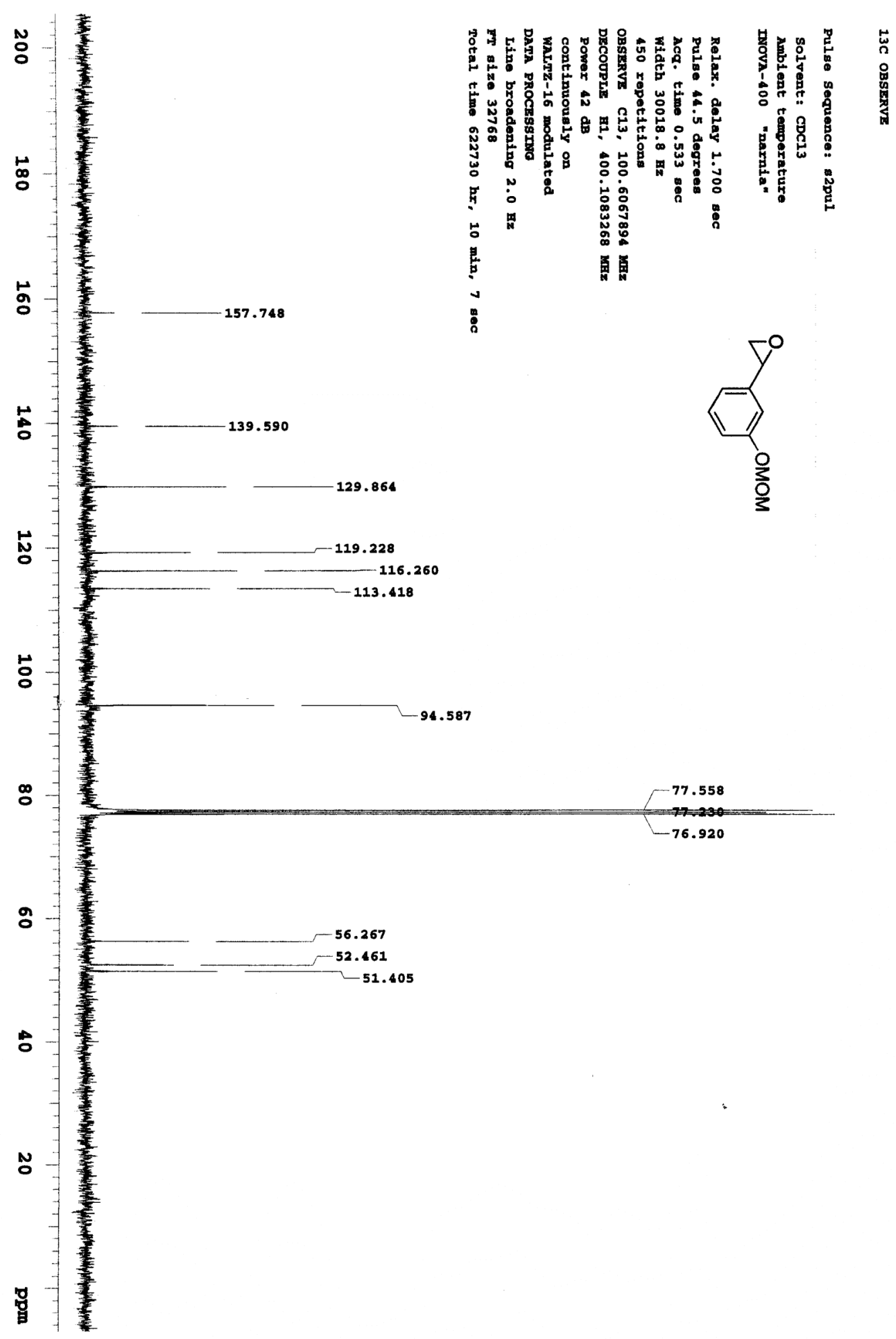




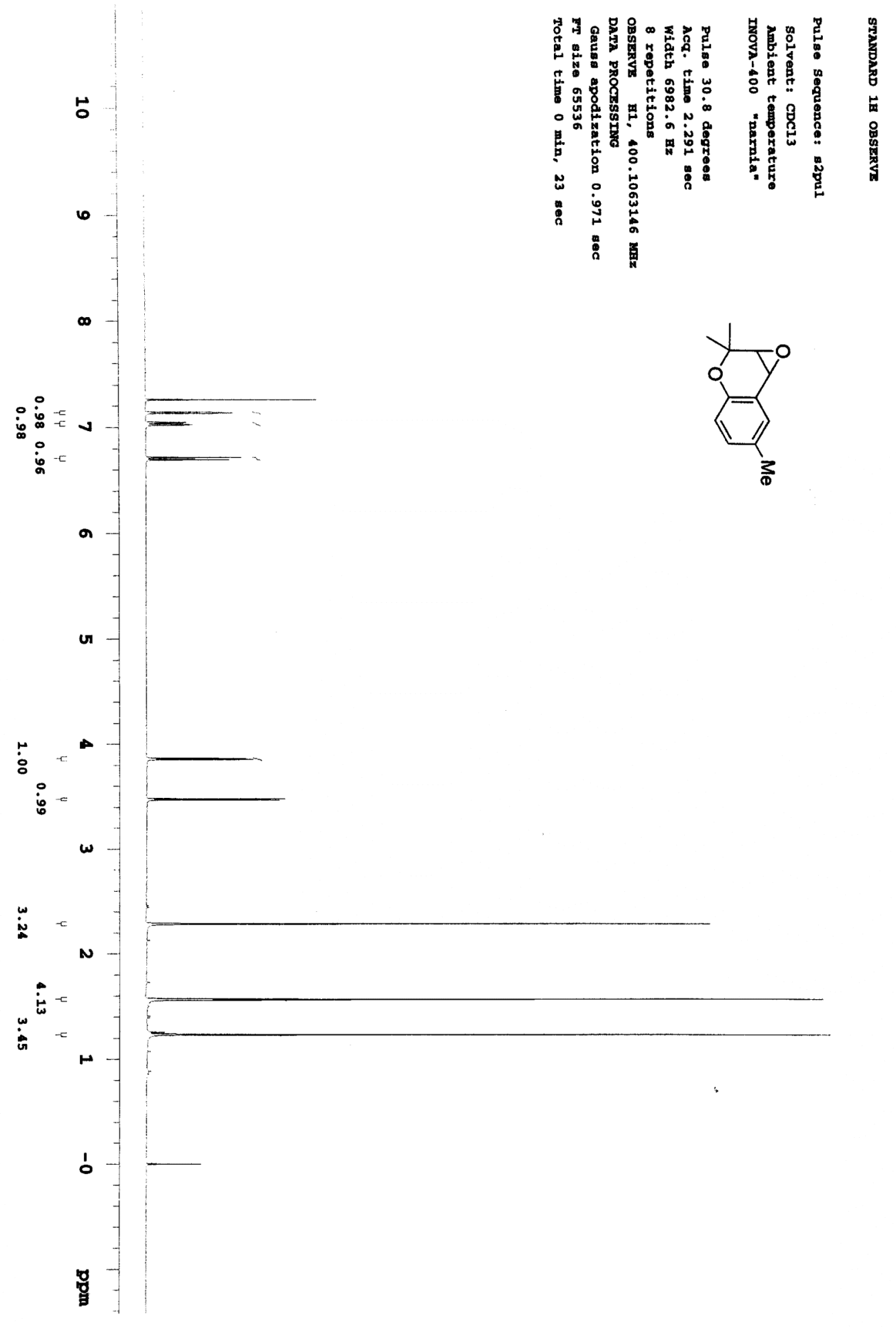




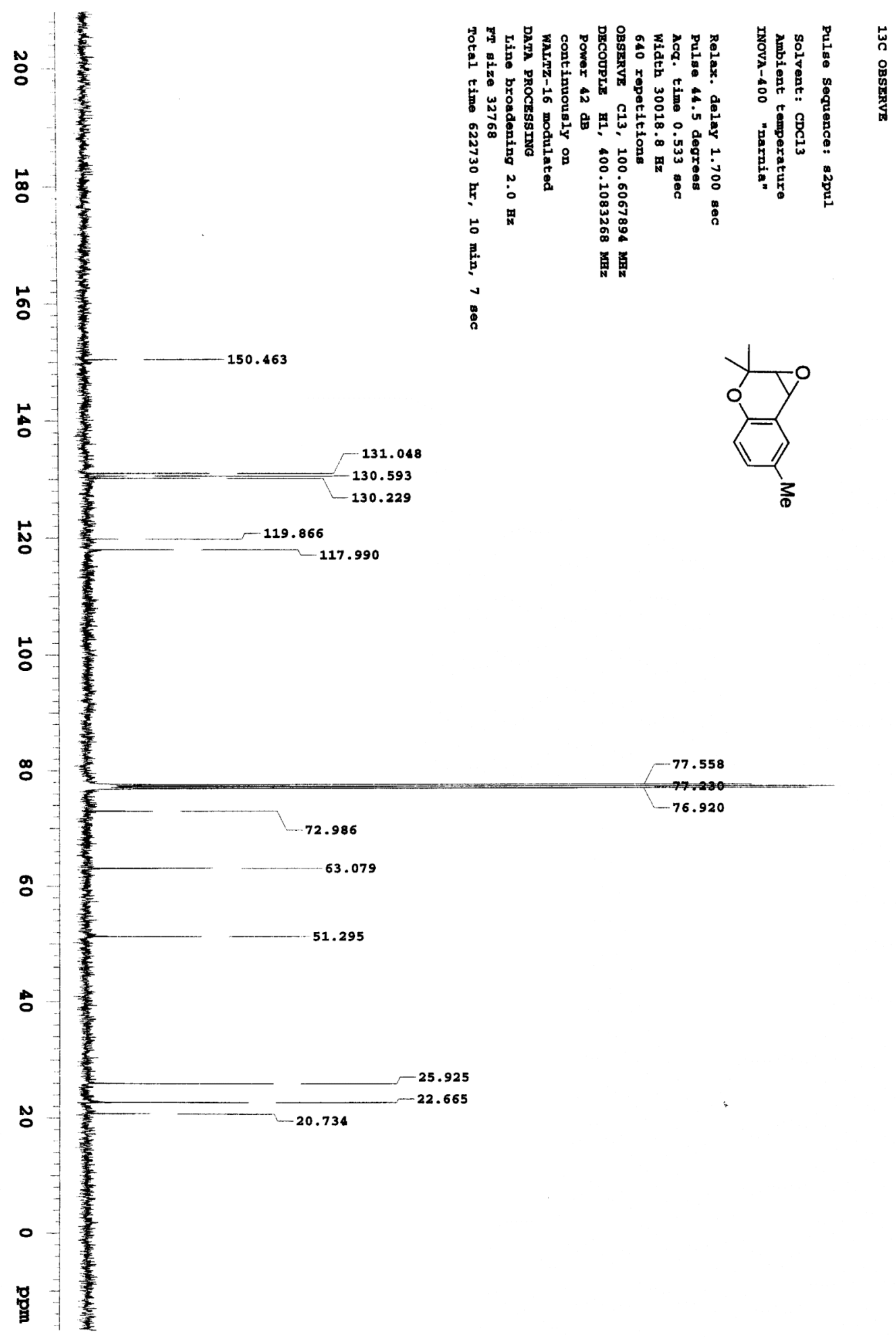




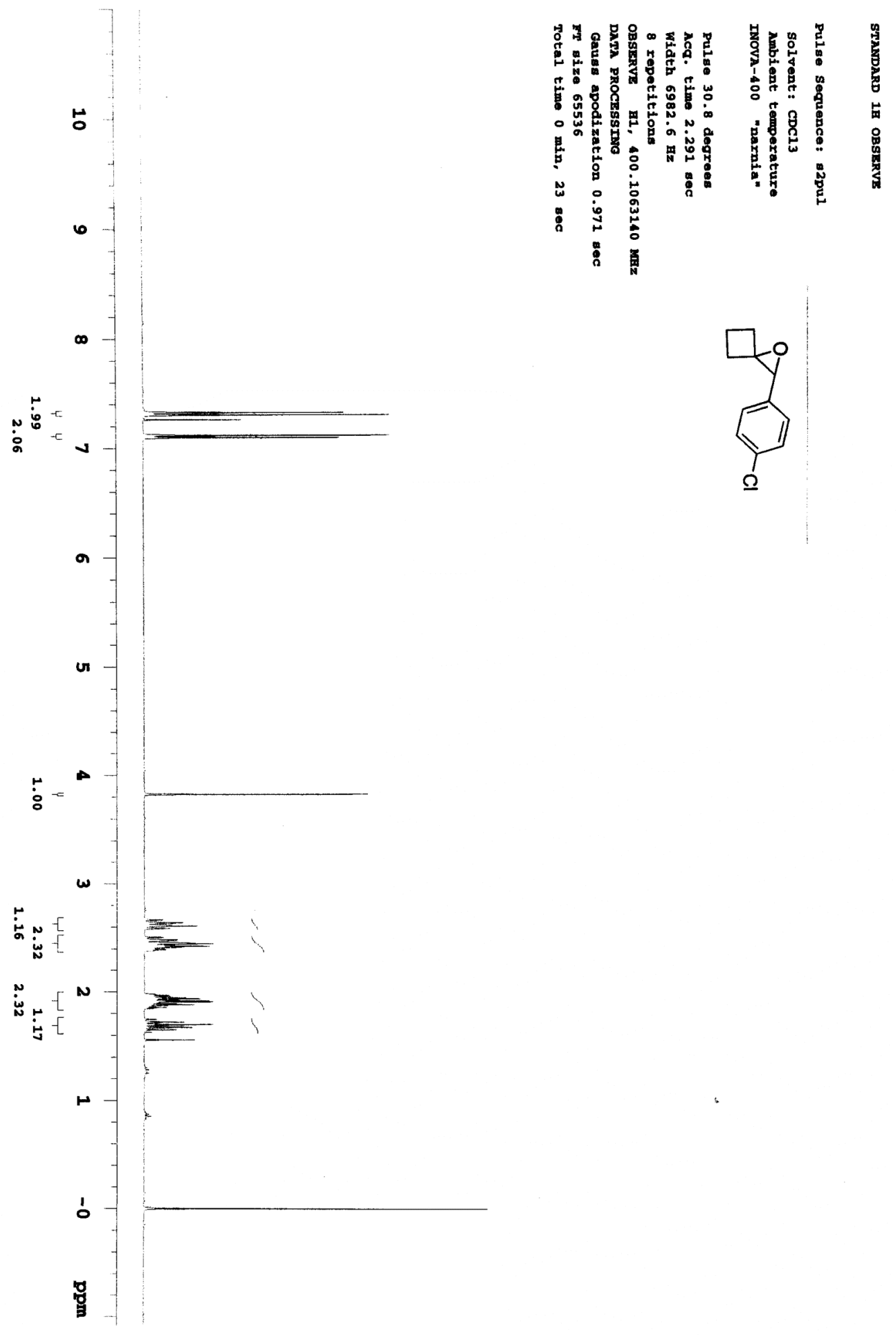




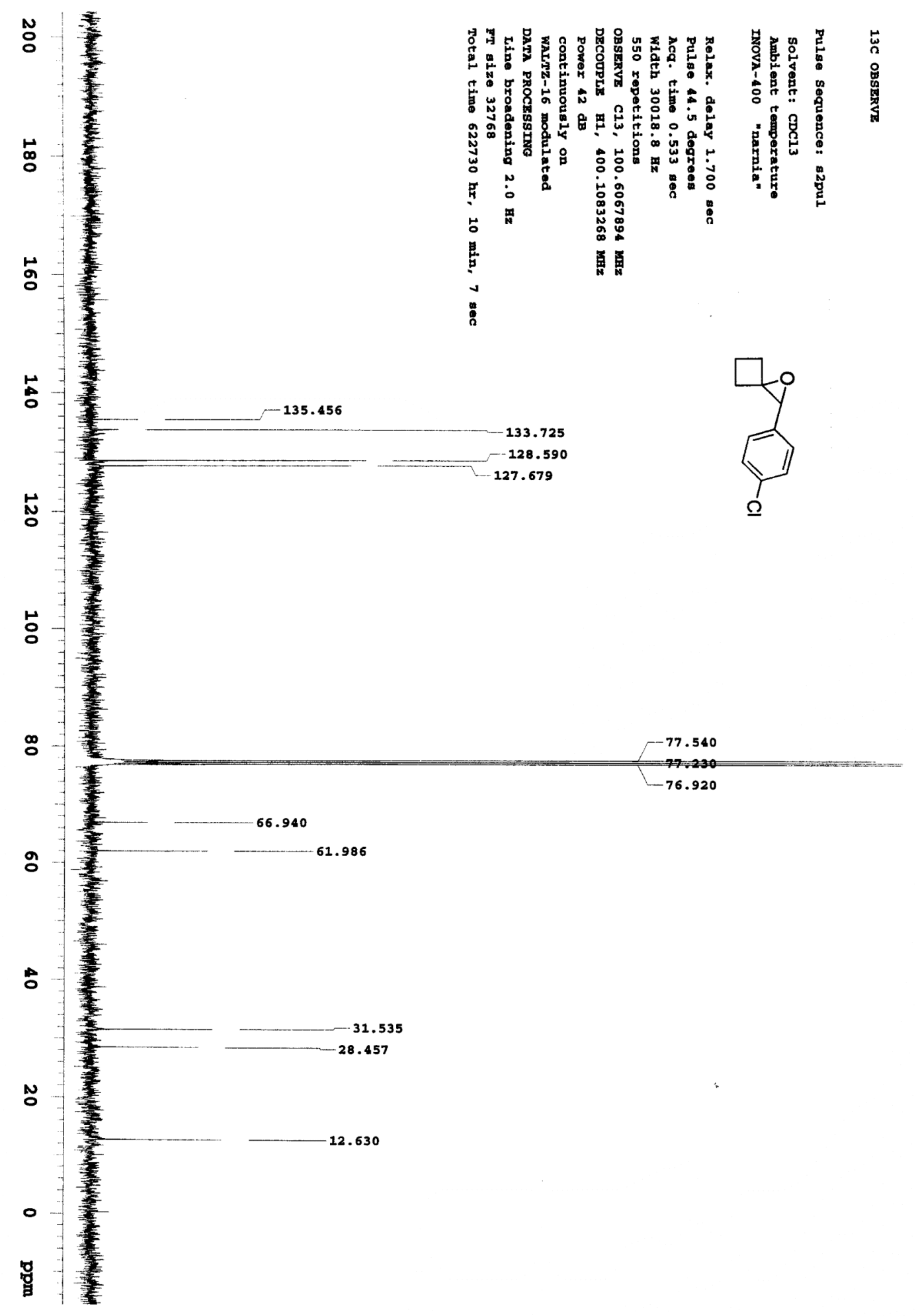




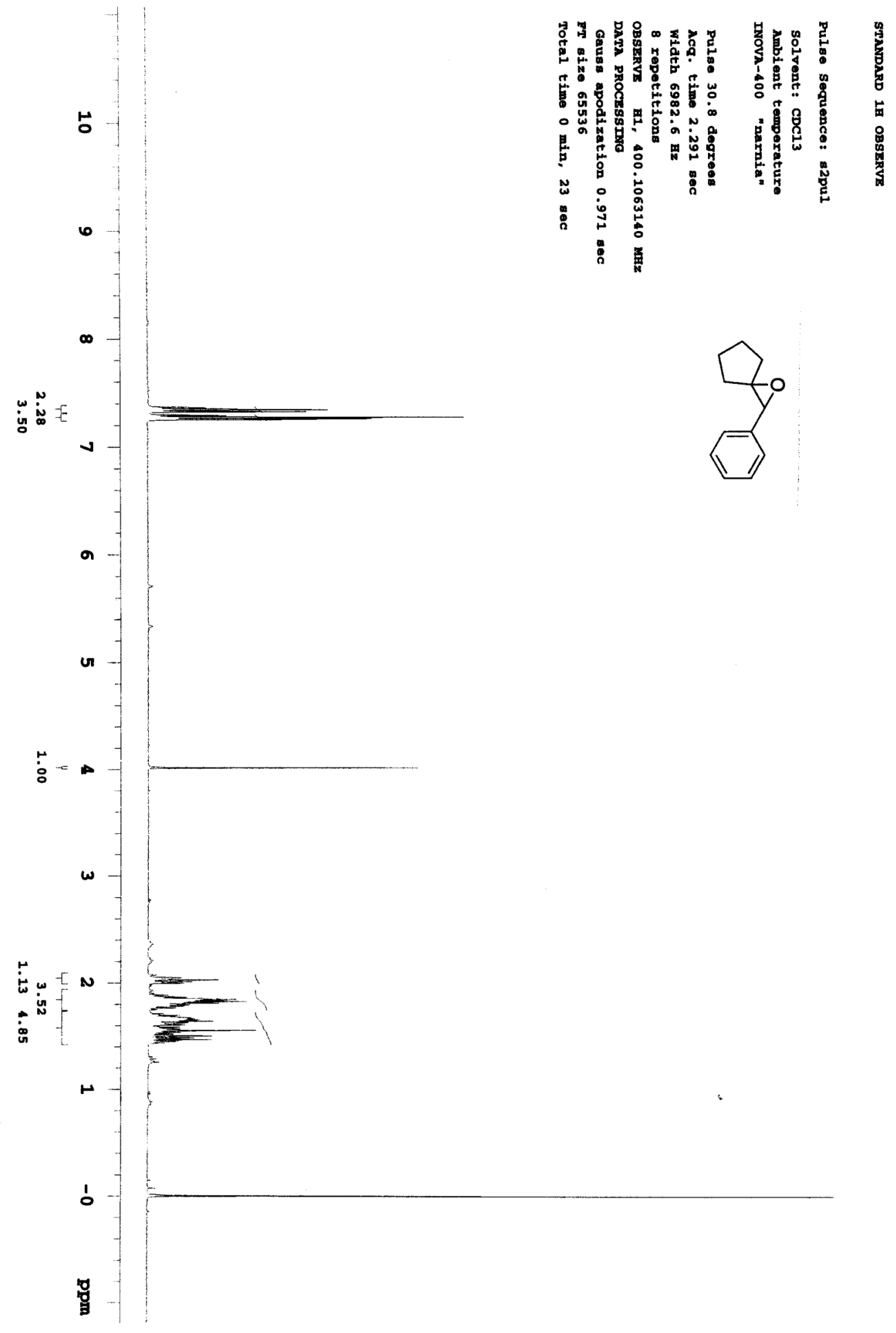




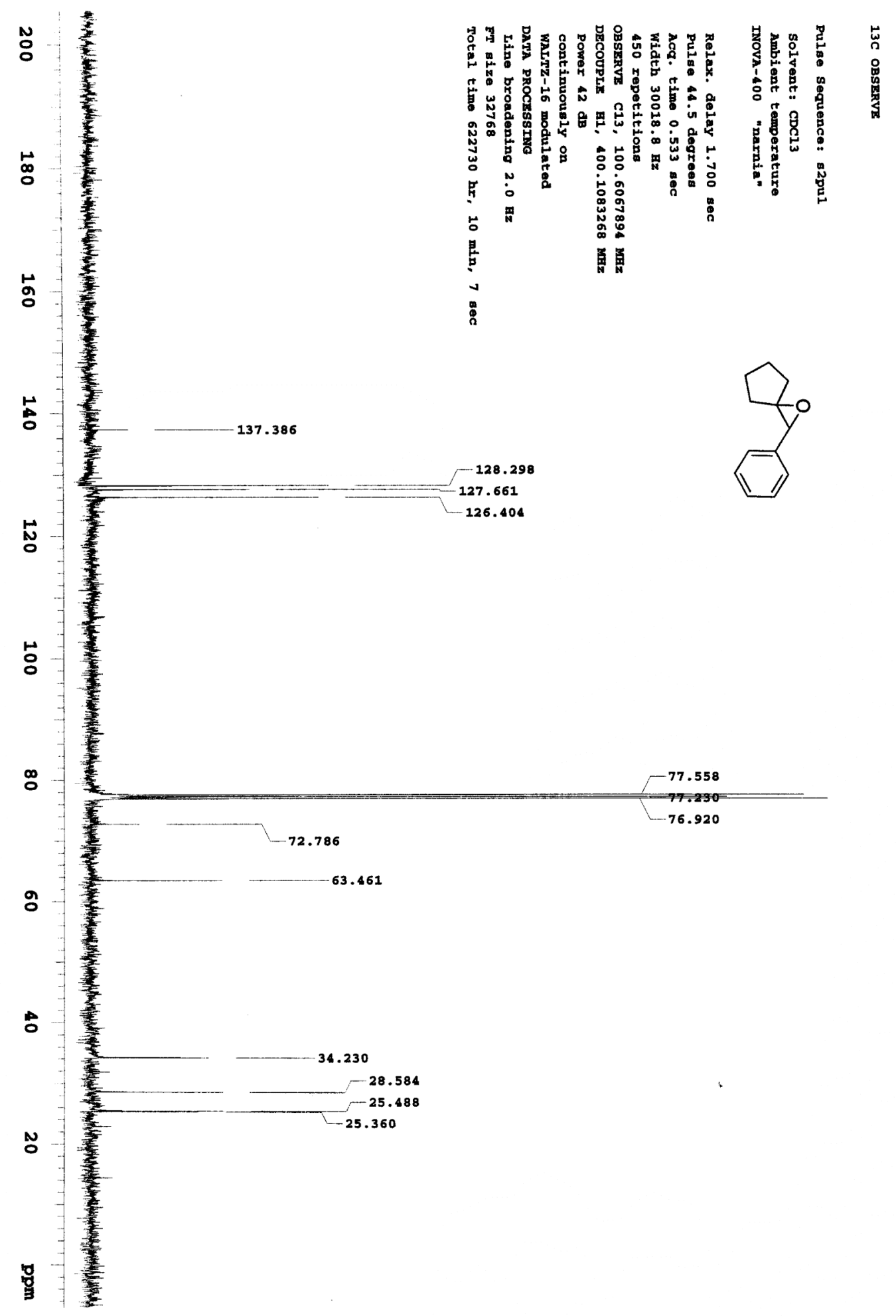




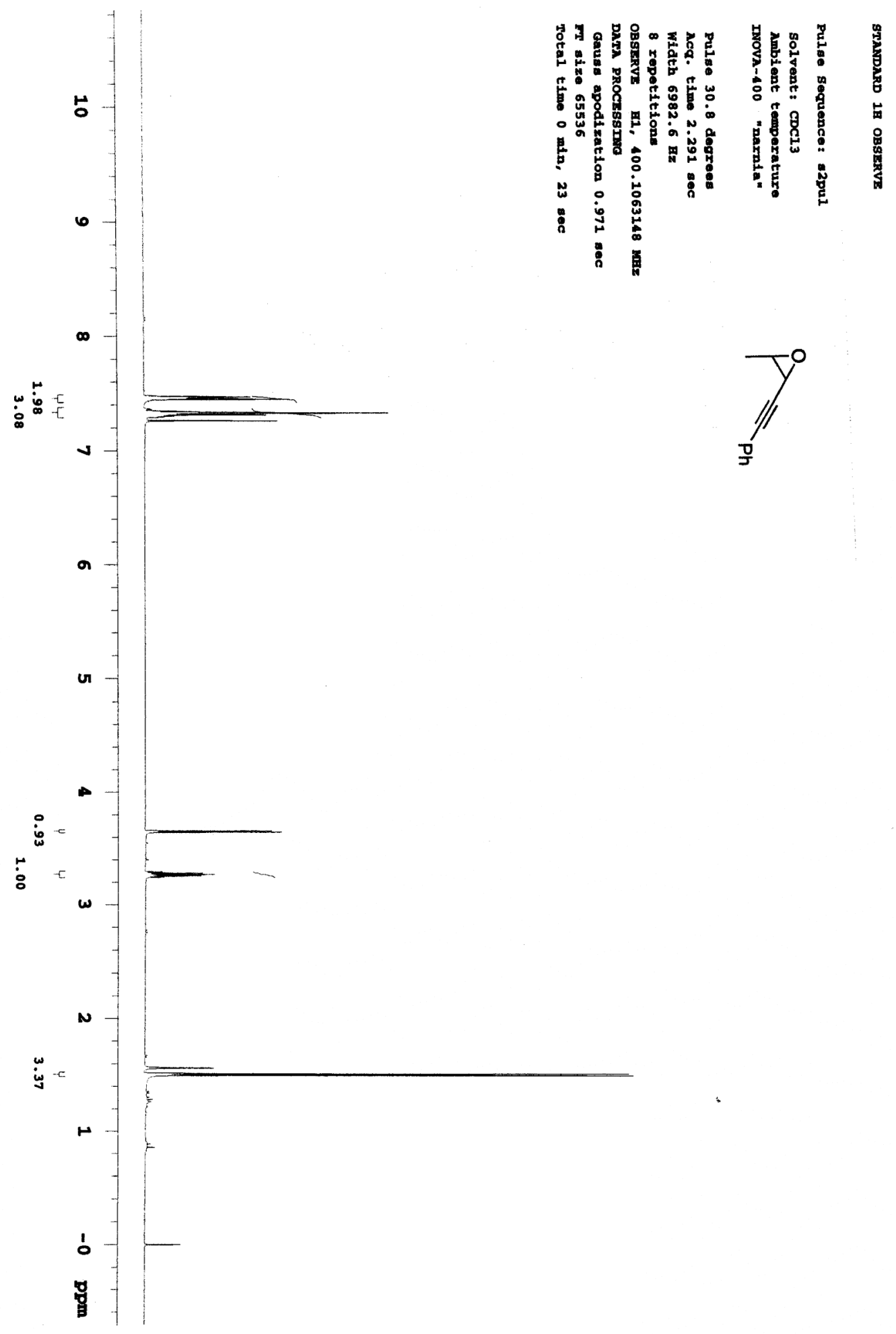




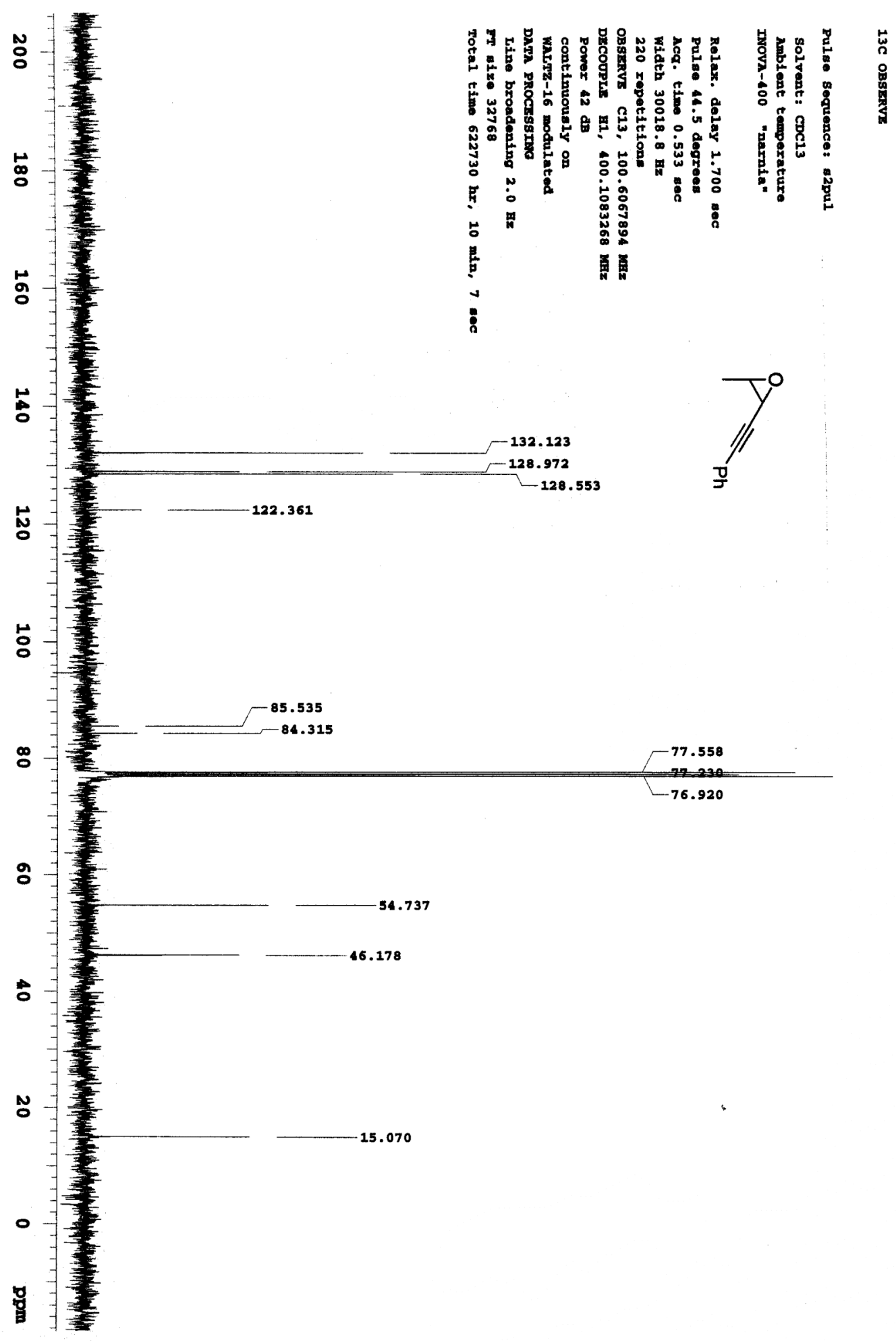



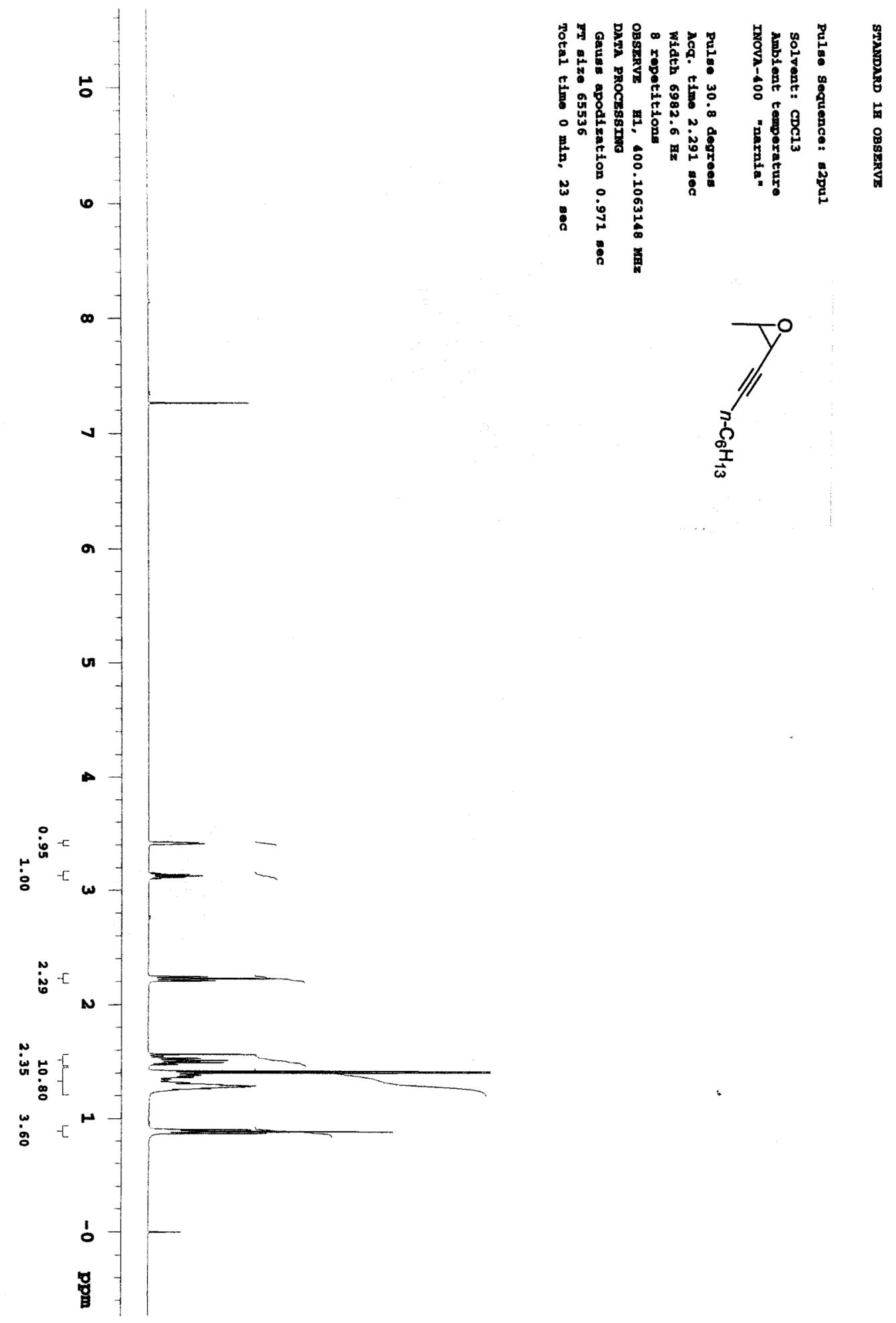


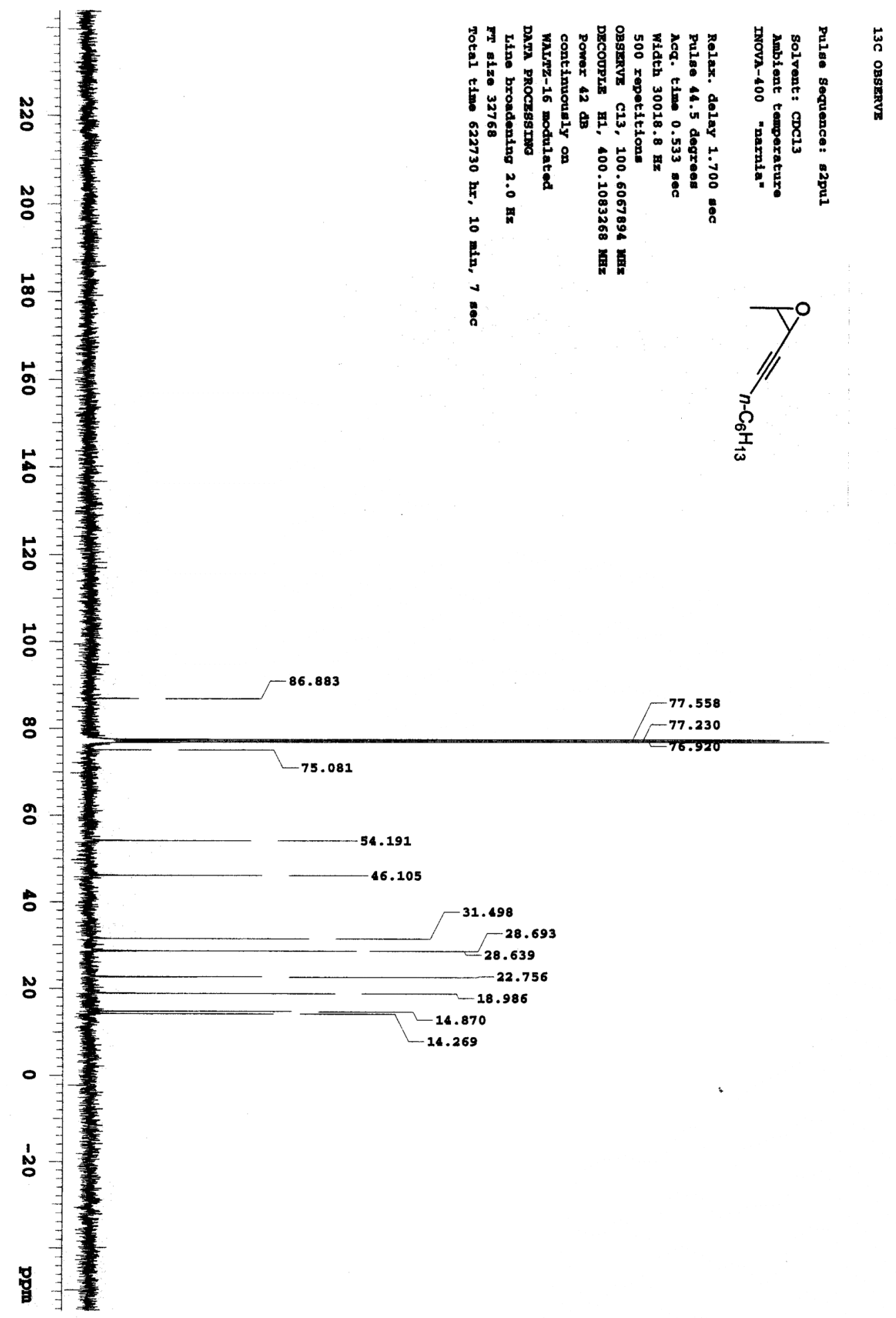




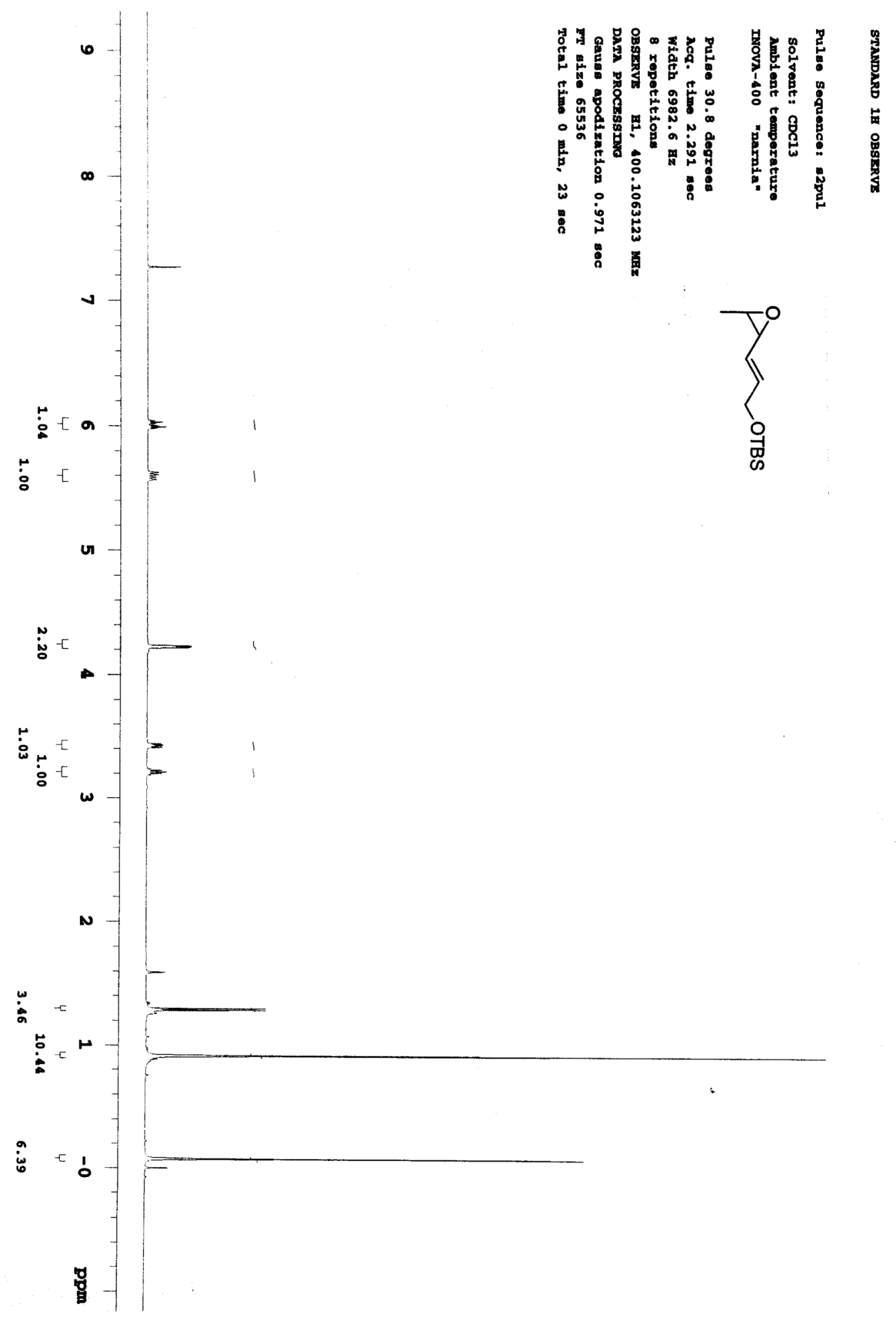




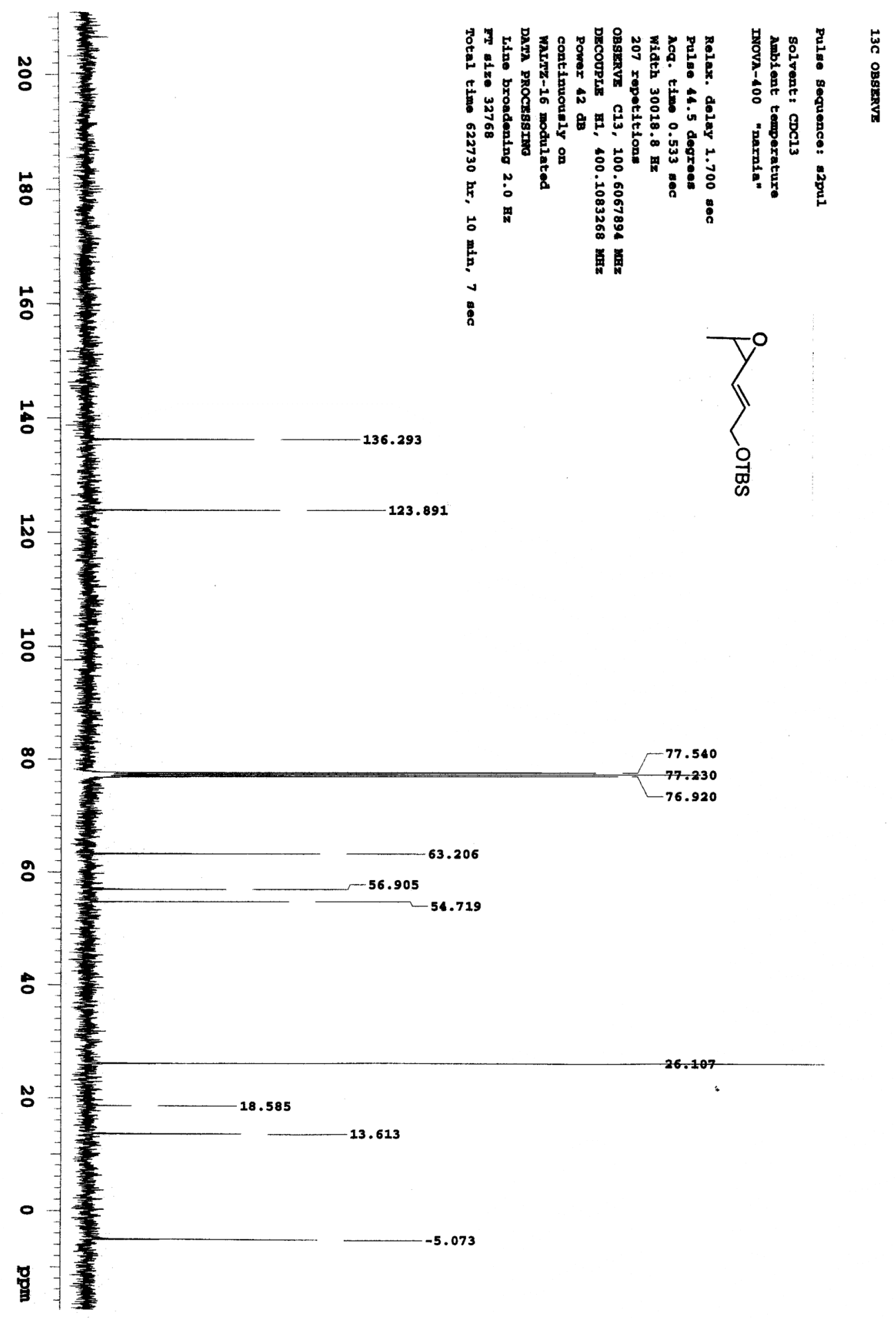

